\title{
Direct Catalytic Synthesis of Enantiopure 5-Substituted Oxazolidinones From Racemic Terminal Epoxides
}

Giuseppe Bartoli, ${ }^{*}$ Marcella Bosco, Armando Carlone, Manuela Locatelli, Paolo Melchiorre,* and Letizia Sambri

Department of Organic Chemistry “A. Mangini”, Alma Mater Studiorum - Bologna University v.le Risorgimento 4, I-40136 Bologna, Italy

e-mail: giuseppe.bartoli@unibo.it; pm@ms.fci.unibo.it

\section{Contents}

General Methods..........................................................................

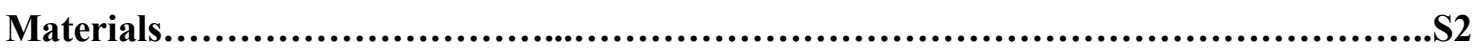

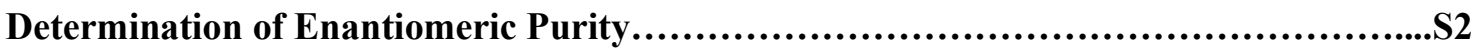

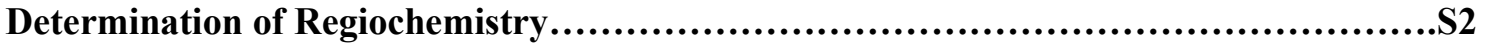

Determination of Absolute Configuration................................................S2

Calculation of conversion $(c)$ and evaluation of selectivity factor $s \ldots \ldots \ldots \ldots \ldots \ldots \ldots \ldots \ldots . . . .53$

Experimental Procedures............................................................... 4

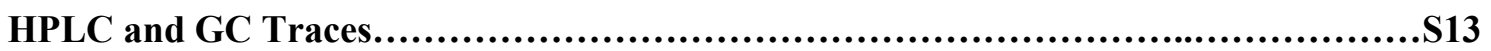

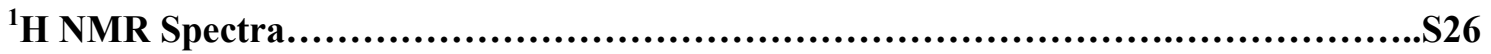

General Methods. The ${ }^{1} \mathrm{H}$ and ${ }^{13} \mathrm{C}$ NMR spectra were recorded at $400 \mathrm{MHz}$ and $100 \mathrm{MHz}$, respectively. The chemical shifts $(\delta)$ for ${ }^{1} \mathrm{H}$ and ${ }^{13} \mathrm{C}$ are given in ppm relative to residual signals of the solvent $\left(\mathrm{CHCl}_{3}\right)$. Coupling constants are given in $\mathrm{Hz}$. Carbon types were determined from DEPT ${ }^{13} \mathrm{C}$ NMR experiments. The following abbreviations are used to indicate the multiplicity: s, singlet; d, doublet; t, triplet; q, quartet; m, multiplet; bs, broad signal. Purification of reaction products was carried out by flash chromatography (FC) on silica gel (230-400 mesh). Melting points are uncorrected. Optical rotations are reported as follows: $[\alpha]_{D}^{\mathrm{rt}}(c$ in $\mathrm{g}$ per $100 \mathrm{~mL}$, solvent). 
Materials. Commercial grade reagents and solvents were used without further purification; otherwise, where necessary, they were purified as recommended. ${ }^{1}$

Racemic epoxides: 1,2-epoxy butane 2a, propylene oxide $\mathbf{2 b}$, 1,2-epoxy-7-octene $\mathbf{2}$ c, isopropyl glycidyl ether $\mathbf{2 f}$, and styrene oxide $\mathbf{2 h}$ were purchased from Aldrich and used as received. $O$ silylated epoxide $2 \mathrm{~g}$ was prepared by protection of racemic glicydol $\left(\mathrm{CH}_{2} \mathrm{Cl}_{2} /\right.$ imidazole / tert-

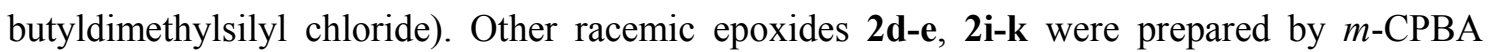
oxidation of the corresponding alkenes. ${ }^{2}$

Urethane 3 was purchased from Aldrich and used as received.

$N$-Boc-2-nitrobenzenesulfonamide $\mathbf{6}$ was prepared according to literature procedure. ${ }^{3}$

Both $(\mathrm{R}, \mathrm{R})$ and $(\mathrm{S}, \mathrm{S})-(\mathrm{salen})-\mathrm{Co}(\mathrm{II})$ complexes $\mathbf{1}$ (Aldrich) are commercially available.

Determination of Enantiomeric Purity. Chiral HPLC analysis was performed on an Agilent 1100-series instrumentation. Daicel Chiralcel OD and Chiralpak AD-H, OB-H or AS-H columns with $\mathrm{i}-\mathrm{PrOH} /$ hexane as the eluent were used.

Chiral GC analysis was performed on a Hewlett-Packard 5890 gas chromatography using a RTBetaDEX-sm chiral column.

The enantiomeric excess (ee) of the products was determined by HPLC analysis on the oxazolidinones $4 \mathbf{h}-\mathbf{k}$ and on the corresponding $N$-benzoyl derivatives for oxazolidinones $4 \mathbf{c}-\mathbf{e}$ and $\mathbf{4 g}$. The enantiomeric excess (ee) of the oxazolidinones $\mathbf{4 a - b}$ and $\mathbf{4 f}$ was determined by GC analysis. HPLC and GC traces were compared to racemic samples prepared with racemic (salen)-Co ${ }^{\mathrm{III}}$ complex as the catalyst.

Determination of Regiochemistry. The regiochemistry of the ring opening reactions was determined by careful GC and NMR analysis of the crude reaction mixtures and by comparison with literature data.

Determination of Absolute Configuration. The measured optical rotation of oxazolidinones $\mathbf{4 b},{ }^{4}$ and $\mathbf{4 h},{ }^{5}$ were compared with the literature value. All other absolute configurations were assigned by analogy.

\footnotetext{
${ }^{1}$ Armarengo, W. L. F.; Perrin, D. D.; In Purification of Laboratory Chemicals, 4th ed.; Butterworth Heinemann: Oxford, 1996.

${ }^{2}$ Moyna, G.; Williams, H. J.; Scott, A. L. Synth. Commun. 1996, 26, 2235.

${ }^{3}$ Fukuyama, T.; Cheung, M.; Kan, T. Synlett 1999, 1301.

${ }^{4}$ Nishiyama, T.; Matsui, S.; Yamada, F. J. Heterocycl. Chem. 1986, 23, 1427.

${ }^{5}$ Matsumura, Y.; Ohishi, T.; Sonoda, C.; Maki, T.; Watanabe, M. Tetrahedron, 1997, 53, 4579.
} 
Calculation of conversion (c) and evaluation of selectivity factor $s .{ }^{6}$ Evaluation of selectivity factor $s$ was accomplished by using the equation: $s=k_{r e l}=\ln [1-c(1+\mathrm{ee})] / \ln [1-c(1-\mathrm{ee})]$, where ee is the enantiomeric excess of the oxazolidinone product and $c$ is the conversion. The conversion was set to equal the isolated yield of the oxazolidinone product, after the sequential one-pot cyclization of the corresponding amino alcohol, so calculated values for $c$ actually represent lower limits: the value for $c$ was calculated by multiplying the isolated yield times 0.476 for entries 1-3 and 5-7 (2.1 equiv of epoxides used) and times 0.454 for entries 4 and 8-11 (2.2 equiv of epoxides used) (Table $\mathrm{S} 1$ ).

Indeed, the $s$ values in table S1 represent just an evaluation of the efficiency of the presented methodology, that stems from the extraordinary level of selectivity of the carbamate-based Aminolytic Kinetic Resolution (AKR) strategy: the exact calculation of the selectivity factors should be done on the amino alcohol products. ${ }^{7}$ The evaluation of the selectivity on the oxazolidinone products should imply that the cyclization of the corresponding aminols proceeds without racemization. While this is true for aliphatic substrates, we have observed that the enantiopurity of the aromatic oxazolidinones is slightly affected by the cyclization step (the corresponding aminols are produced in enantiomerically pure form, ee $>99 \%$ )

Table S1. Evaluation of $s$ Values for the Direct Conversion of Terminal Epoxides to Enantiopure Oxazolidinones 4.

\begin{tabular}{ccccccc}
\hline Entry & $\mathrm{R}$ & product & yield & $c$ & ee & $s$ \\
\hline 1 & $\mathrm{Et}$ & $\mathbf{4 a}$ & 0.94 & 0.447 & 99.9 & $>3000$ \\
2 & $\mathrm{Me}$ & $\mathbf{4 b}$ & 0.97 & 0.471 & 99.9 & $>3000$ \\
3 & $\left(\mathrm{CH}_{2}\right)_{4} \mathrm{CH}=\mathrm{CH}_{2}$ & $\mathbf{4 c}$ & 0.95 & 0.452 & 99.7 & 1715 \\
4 & $c-\mathrm{C}_{6} \mathrm{H}_{11}$ & $\mathbf{4 d}$ & 0.97 & 0.461 & 99.2 & 680 \\
5 & $\mathrm{CH}_{2} \mathrm{Ph}$ & $\mathbf{4 e}$ & 0.98 & 0.466 & 99.5 & 670 \\
6 & $\mathrm{CH}_{2} \mathrm{O}(i-\mathrm{Pr})$ & $\mathbf{4 f}$ & 0.90 & 0.428 & 99.5 & 900 \\
7 & $\mathrm{CH}_{2} \mathrm{OTBS}$ & $\mathbf{4 g}$ & 0.92 & 0.438 & 99.0 & 465 \\
8 & $\mathrm{Ph}$ & $\mathbf{4 h}$ & 0.90 & 0.428 & 99.2 & 560 \\
9 & $4-\mathrm{ClPh}$ & $\mathbf{4 i}$ & 0.98 & 0.466 & 98.7 & 428 \\
10 & $3-\mathrm{ClPh}$ & $\mathbf{4 j}$ & 0.97 & 0.461 & 96.5 & 145 \\
11 & $3-\mathrm{NO}_{2} \mathrm{Ph}$ & $\mathbf{4 k}$ & 0.94 & 0.447 & 97.5 & 190
\end{tabular}

Considering the extraordinarily levels of selectivities $(s>145)$ observed, the relative magnitude of $s$ factors can provide useful guidelines of evaluation of the presented strategy, but the absolute values for the best substrates ( $s$ exceeding 3000) are certainly lacking precision.

\footnotetext{
${ }^{6}$ Kagan, H. B.; Fiaud, J. C. In Topics in Stereochemistry; Allinger, N. L.; Eliel, E. L. Eds.; Interscience: New York, 1987; Vol. 14, p 249.

7 For an exact calculation of the selectivity of the AKR, see: Bartoli, G.; Bosco, M.; Carlone, A.; Locatelli, M.; Melchiorre, P.; Sambri, L. Org. Lett. 2004, 6, 3973 and Supporting Information therein.
} 


\section{Experimental Procedures.}

General Procedure for the $\boldsymbol{N}$-Benzoylation of Oxazolidinones $4 \mathrm{c}-\mathrm{e}$ and $\mathbf{4 g}$. Under an inert atmosphere, pure oxazolidinones $\mathbf{4 c - e}, \mathbf{g}$ were dissolved in THF followed by the addition of benzoyl chloride (1.3 equiv) and $\mathrm{NaH}\left(1.1\right.$ equiv) at $0^{\circ} \mathrm{C}$. The reaction mixture was allowed to rise to room temperature, stirred for 3 hours and quenched with $\mathrm{NaHCO}_{3}$, extracted with $\mathrm{Et}_{2} \mathrm{O}$ and dried over anhydrous $\mathrm{MgSO}_{4}$. Purification by flash chromatography (hexane/AcOEt 9/1) afforded pure $N$-benzoyl oxazolidinones.

General Procedure for the Direct Conversion of Racemic Terminal Epoxides to Enantiopure Oxazolidinones. All the reactions were carried out in undistilled solvent without any precautions to exclude water. In an ordinary test tube equipped with a magnetic stirring bar, the (salen)-Co ${ }^{\mathrm{II}}$ complex 1 ( $\mathrm{x}$ mol\% relative to racemic epoxide, $\mathrm{x}=1.5-5$ ) was dissolved in 0.15 $\mathrm{mL}$ of TBME. After addition of the oxidizing agent ( $p$-nitrobenzoic acid, $2 \mathrm{x}$ mol\%), the tube was closed with a rubber stopper and the mixture was stirred at room temperature over which time the color changed from orange-red to dark brown. Then the urethane 3 (44.5 mg, 0.5 mmol) was added. After 5 minutes stirring, racemic epoxide $2(1.05 \mathrm{mmol}$ or $1.1 \mathrm{mmol})$ was added and stirring was continued at room temperature till the completion of the AKR step, as indicated by TLC analysis, at which time $5 \mathrm{~mL}$ of THF and $1 \mathrm{mmol}$ of $\mathrm{NaH}(24 \mathrm{mg})$ were added. After $3 \mathrm{~h}$ stirring, the crude reaction mixture was quenched with $\mathrm{NH}_{4} \mathrm{Cl}$ and diluted with $\mathrm{Et}_{2} \mathrm{O}$. The organic layer was washed with brine and dried over $\mathrm{Na}_{2} \mathrm{SO}_{4}$. After filtration, solvent was removed in vacuo, and the residue was purified by flash chromatography to yield the 5substituted oxazolidinone 4a-k in almost enantiomerically pure form.
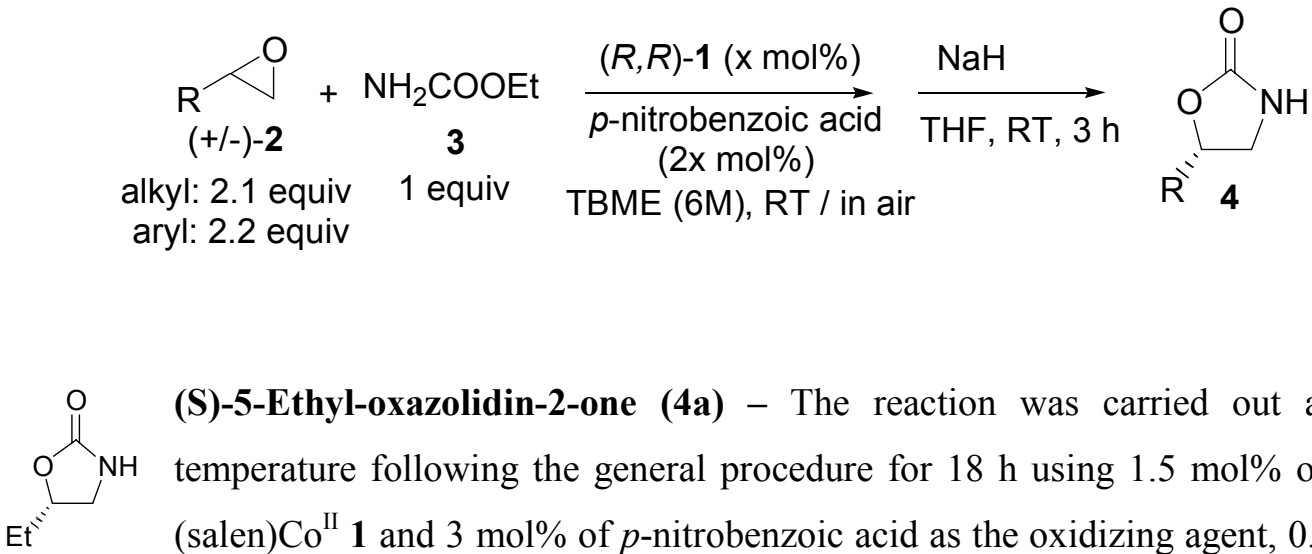

(S)-5-Ethyl-oxazolidin-2-one (4a) - The reaction was carried out at room temperature following the general procedure for $18 \mathrm{~h}$ using $1.5 \mathrm{~mol} \%$ of $(R, R)$ (salen)Co ${ }^{\text {II }} 1$ and 3 mol\% of $p$-nitrobenzoic acid as the oxidizing agent, $0.5 \mathrm{mmol}$ of urethane 3 and $1.05 \mathrm{mmol}$ of racemic 1,2-epoxy butane 2a. After the cyclization step, the title compound was isolated by column chromatography (DCM/AcOEt 6/4) as a white solid in $94 \%$ yield; melting point: $51-53{ }^{\circ} \mathrm{C}$. The ee was determined by $\mathrm{GC}$ analysis (isotherm $150{ }^{\circ} \mathrm{C} ; \tau_{\mathrm{R}}=$ $\left.19.6 \mathrm{~min} ; \tau_{\mathrm{S}}=20.1 \mathrm{~min}\right) .[\alpha]_{\mathrm{D}}^{\mathrm{rt}}=-14.7^{\circ}\left(c=1, \mathrm{CHCl}_{3}, 99.9 \%\right.$ ee). ESI-MS $m / z 114[\mathrm{M}-\mathrm{H}]^{-} .{ }^{1} \mathrm{H}$ $\operatorname{NMR}\left(\mathrm{CDCl}_{3}\right): \delta=1.01(\mathrm{t}, 3 \mathrm{H}, J=7.5), 1.65-1.87(\mathrm{~m}, 2 \mathrm{H}), 3.25(\mathrm{ddd}, J=1.2,7.2,8.4,1 \mathrm{H})$, $3.66(\mathrm{ddd}, J=1.2,8.4,8.4,1 \mathrm{H}), 4.55-4.62(\mathrm{~m}, 1 \mathrm{H}), 5.30(\mathrm{bs}, 1 \mathrm{H}, \mathrm{NH}),{ }^{13} \mathrm{C} \mathrm{NMR}\left(\mathrm{CDCl}_{3}\right): \delta=$ $8.7\left(\mathrm{CH}_{3}\right), 27.8\left(\mathrm{CH}_{2}\right), 45.5\left(\mathrm{CH}_{2}\right), 78.2(\mathrm{CH}), 160.3(\mathrm{C})$. 


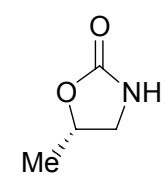

(S)-5-Methyl-oxazolidin-2-one (4b) ${ }^{8}$ - The reaction was carried out following the general procedure at room temperature for $18 \mathrm{~h}$ using $1.5 \mathrm{~mol} \%$ of $(R, R)$ (salen)Co ${ }^{\text {II }} \mathbf{1}$ and $3 \mathrm{~mol} \%$ of $p$-nitrobenzoic acid as the oxidizing agent, $0.5 \mathrm{mmol}$ of urethane $\mathbf{3}$ and $1.05 \mathrm{mmol}$ of racemic propylene oxide $\mathbf{2} \mathbf{b}$. After the cyclization step, the title compound was isolated by column chromatography (DCM/AcOEt 3/7) as a colourless oil in $97 \%$ yield. The ee was determined by GC analysis (isotherm $145{ }^{\circ} \mathrm{C} ; \tau_{\mathrm{R}}=15.1$ $\left.\min ; \tau_{\mathrm{S}}=15.5 \mathrm{~min}\right) .[\alpha]^{\mathrm{rt}}=-8.9^{\circ}\left(c=1.0, \mathrm{CHCl}_{3}, 99.9 \%\right.$ ee $)$, lit. $^{4}[\alpha]^{\mathrm{rt}}{ }_{\mathrm{D}}=-20.9^{\circ},(\mathrm{S})-4 \mathbf{b}$. ESI-MS $m / z 102[\mathrm{M}+\mathrm{H}]^{+}, 124[\mathrm{M}+\mathrm{Na}]^{+} .{ }^{1} \mathrm{H} \mathrm{NMR}\left(\mathrm{CDCl}_{3}\right): \delta=1.45(\mathrm{~d}, 3 \mathrm{H}, J=6.2), 3.19$ (ddd, $J=0.8$, 7.2, 8.4, 1H), 3.69 (ddd, $J=0.8,8.4,8.4,1 \mathrm{H}), 4.73-4.81(\mathrm{~m}, 1 \mathrm{H}), 5.58(\mathrm{bs}, 1 \mathrm{H}, \mathrm{NH}) ;{ }^{13} \mathrm{C} \mathrm{NMR}$ $\left(\mathrm{CDCl}_{3}\right): \delta=20.8\left(\mathrm{CH}_{3}\right), 47.7\left(\mathrm{CH}_{2}\right), 73.8(\mathrm{CH}), 160.5(\mathrm{C})$.

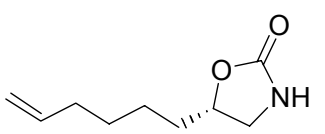

(S)-5-Hex-5-enyl-oxazolidin-2-one (4c) - The reaction was carried out following the general procedure at room temperature for $18 \mathrm{~h}$ using $1.5 \mathrm{~mol} \%$ of $(R, R)$-(salen)Co ${ }^{\mathrm{II}} \mathbf{1}$ and $3 \mathrm{~mol} \%$ of $p$-nitrobenzoic acid as the oxidizing agent, $0.5 \mathrm{mmol}$ of urethane 3 and $1.05 \mathrm{mmol}$ of racemic 1,2-epoxy-7octene 2c. After the cyclization step, the title compound was isolated by column chromatography (DCM/AcOEt $8 / 2$ ) as a white solid in $95 \%$ yield; melting point: $63-65{ }^{\circ} \mathrm{C}$. The ee was determined by HPLC analysis on the corresponding $N$-benzoyl derivative, prepared following the described general procedure, using a Chiralpak AD-H column (90/10 hexane/ $i$ PrOH; flow rate $\left.0.75 \mathrm{~mL} / \mathrm{min} ; \tau_{\mathrm{R}}=20.4 \mathrm{~min} ; \tau_{\mathrm{S}}=24.5 \mathrm{~min}\right) .[\alpha]_{\mathrm{D}}^{\mathrm{rt}}=-23.0^{\circ}\left(c=2.0, \mathrm{CHCl}_{3}\right.$, 99.7\% ee). ESI-MS $m / z 168$ [M-H] ${ }^{-1}{ }^{1} \mathrm{H}$ NMR $\left(\mathrm{CDCl}_{3}\right): \delta=1.32-1.55(\mathrm{~m}, 4 \mathrm{H}), 1.59-1.71(\mathrm{~m}$, $1 \mathrm{H}), 1.76-1.86(\mathrm{~m}, 1 \mathrm{H}), 2.04-2.10(\mathrm{~m}, 2 \mathrm{H}), 3.23(\mathrm{ddd}, J=0.8,7.2,8.4,1 \mathrm{H}), 3.66(\mathrm{ddd}, J=0.8$, 8.4, 8.4, 1H), 4.60-4.67 (m, 1H), 4.94-5.04 (m, 2H), $5.13(\mathrm{bs}, 1 \mathrm{H}, \mathrm{NH}), 5.74-5.84(\mathrm{~m}, 1 \mathrm{H}) ;{ }^{13} \mathrm{C}$ $\operatorname{NMR}\left(\mathrm{CDCl}_{3}\right): \delta=24.0\left(\mathrm{CH}_{2}\right), 28.4\left(\mathrm{CH}_{2}\right), 33.4\left(\mathrm{CH}_{2}\right), 34.7\left(\mathrm{CH}_{2}\right), 45.9\left(\mathrm{CH}_{2}\right), 77.1(\mathrm{CH})$, $114.7(\mathrm{CH}), 138.3\left(\mathrm{CH}_{2}\right), 160.2(\mathrm{C})$.

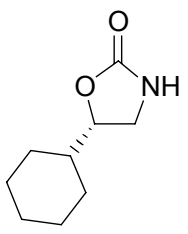

(S)-5-Cyclohexyl-oxazolidin-2-one (4d) - The reaction was carried out following the general procedure at room temperature for $40 \mathrm{~h}$ using $4 \mathrm{~mol} \%$ of $(R, R)$-(salen) $\mathrm{Co}^{\mathrm{II}} \mathbf{1}, 8 \mathrm{~mol} \%$ of $p$-nitrobenzoic acid as the additive, $0.5 \mathrm{mmol}$ of urethane $\mathbf{3}$ and $1.1 \mathrm{mmol}$ of racemic epoxide $\mathbf{2 d}$. After the cyclization step, the title compound was isolated by column chromatography (DCM/AcOEt 7/3) as a white solid in 97\% yield; melting point: $130-131{ }^{\circ} \mathrm{C}$. The ee was determined by HPLC analysis on the corresponding $N$-benzoyl derivative, prepared following the described general procedure, using a Chiralpak AD-H column $\left(85 / 15\right.$ hexane $/ i-\mathrm{PrOH}$; flow rate $0.75 \mathrm{~mL} / \mathrm{min} ; \tau_{\mathrm{R}}=18.7 \mathrm{~min} ; \tau_{\mathrm{S}}=$ $28.2 \mathrm{~min}) .[\alpha]^{\mathrm{rt}} \mathrm{D}=-35.8\left(c=1.0, \mathrm{CHCl}_{3}, 99.2 \%\right.$ ee). ESI-MS $m / z 170[\mathrm{M}+\mathrm{H}]^{+}, 192[\mathrm{M}+\mathrm{Na}]^{+} .{ }^{1} \mathrm{H}$

\footnotetext{
${ }^{8}$ Gabriel, B.; Mancuso, R.; Salerno, G.; Costa, M. J. Org. Chem. 2003, 68, 601.
} 
$\operatorname{NMR}\left(\mathrm{CDCl}_{3}\right): \delta=1.00-1.30(\mathrm{~m}, 5 \mathrm{H}), 1.58-1.80(\mathrm{~m}, 5 \mathrm{H}), 1.93-1.96(\mathrm{~m}, 1 \mathrm{H}), 3.32-3.36(\mathrm{~m}$, $1 \mathrm{H}), 3.58(\mathrm{ddd}, J=0.8,8.4,8.4,1 \mathrm{H}), 4.33-4.39(\mathrm{~m}, 1 \mathrm{H}) 5.05(\mathrm{bs}, 1 \mathrm{H}, \mathrm{NH}) ;{ }^{13} \mathrm{C} \mathrm{NMR}\left(\mathrm{CDCl}_{3}\right)$ : $\delta=25.3\left(\mathrm{CH}_{2}\right), 25.5\left(\mathrm{CH}_{2}\right), 26.1\left(\mathrm{CH}_{2}\right), 27.3\left(\mathrm{CH}_{2}\right), 27.9\left(\mathrm{CH}_{2}\right), 42.0(\mathrm{CH}), 43.9\left(\mathrm{CH}_{2}\right), 81.0$ $(\mathrm{CH}), 160.4(\mathrm{C})$.

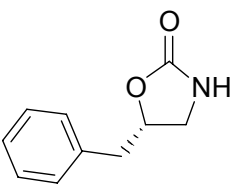

(S)-5-Benzyl-oxazolidin-2-one (4e) - The reaction was carried out following the general procedure at room temperature for $40 \mathrm{~h}$ using $4 \mathrm{~mol} \%$ of $(R, R)$-(salen) $\mathrm{Co}^{\mathrm{II}} \mathbf{1}, 8 \mathrm{~mol} \%$ of $p$-nitrobenzoic acid as the additive, 0.5 mmol of urethane 3 and $1.05 \mathrm{mmol}$ of racemic epoxide 2e. After the cyclization step, the title compound was isolated by column chromatography (DCM/AcOEt 65/35) as a white solid in 98\% yield; melting point: $107-109{ }^{\circ} \mathrm{C}$. The ee was determined by HPLC analysis on the corresponding $N$-benzoyl derivative, prepared following the described general procedure, using a Chiralpak AS-H column $\left(80 / 20\right.$ hexane $/ i-\mathrm{PrOH}$; flow rate $0.75 \mathrm{~mL} / \mathrm{min} ; \tau_{\mathrm{R}}=27.6 \mathrm{~min} ; \tau_{\mathrm{S}}=$ $37.2 \mathrm{~min}) .[\alpha]_{\mathrm{D}}^{\mathrm{rt}}=-20.2^{\circ}\left(c=0.9, \mathrm{CHCl}_{3}, 99.5 \%\right.$ ee $)$. ESI-MS $m / z 176[\mathrm{M}-\mathrm{H}]^{-} .{ }^{1} \mathrm{H}$ NMR $\left(\mathrm{CDCl}_{3}\right): \delta=2.94(\mathrm{dd}, J=6.8,14.0,1 \mathrm{H}), 3.15(\mathrm{dd}, J=6.0,14.0,1 \mathrm{H}), 3.33(\mathrm{ddd}, J=0.8,7.2$, 8.4, 1H), 3.58 (ddd, $J=0.8,8.4,8.4,1 \mathrm{H}), 4.83-4.90$ (m, 1H), 5.59 (bs, 1H, NH), 7.21-7.34 (m, $5 \mathrm{H}) ;{ }^{13} \mathrm{C} \mathrm{NMR}\left(\mathrm{CDCl}_{3}\right): \delta=40.6\left(\mathrm{CH}_{2}\right), 45.0\left(\mathrm{CH}_{2}\right), 77.1(\mathrm{CH}), 127.1(\mathrm{CH}), 128.7(\mathrm{CH}, 2)$, $129.3(\mathrm{CH}, 2), 135.2(\mathrm{C}), 159.8(\mathrm{C})$.

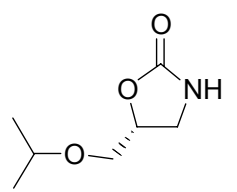

(R)-5-Isopropoxymethyl-oxazolidin-2-one (4f) - The reaction was carried out following the general procedure at room temperature for $24 \mathrm{~h}$ using 2 mol\% of $(R, R)$-(salen)Co ${ }^{\mathrm{II}} \mathbf{1}$ and $4 \mathrm{~mol} \%$ of $p$-nitrobenzoic acid as the oxidizing agent, $0.5 \mathrm{mmol}$ of urethane $\mathbf{3}$ and $1.05 \mathrm{mmol}$ of racemic isopropyl glycidyl ether $\mathbf{2 f}$. After the cyclization step, the title compound was isolated by column chromatography (DCM/AcOEt 6/4) as a colourless oil in $90 \%$ yield. The ee was determined by GC analysis (isotherm $\left.170{ }^{\circ} \mathrm{C} ; \tau_{\mathrm{S}}=19.6 \mathrm{~min} ; \tau_{\mathrm{R}}=20.4 \mathrm{~min}\right)$. $[\alpha]^{\mathrm{rt}}=-7.6^{\circ}\left(c=1.8, \mathrm{CHCl}_{3}, 99.5 \%\right.$ ee). ESIMS $m / z 158[\mathrm{M}-\mathrm{H}]^{-} .{ }^{1} \mathrm{H}$ NMR $\left(\mathrm{CDCl}_{3}\right): 1.16(\mathrm{~d}, 6 \mathrm{H}, J=6.15), 3.45-3.50(\mathrm{~m}, 1 \mathrm{H}), 3.55-3.70$ (m, $4 \mathrm{H}), 4.69-4.75(\mathrm{~m}, 1 \mathrm{H}), 5.31(\mathrm{bs}, 1 \mathrm{H}, \mathrm{NH}) ;{ }^{13} \mathrm{C} \mathrm{NMR}\left(\mathrm{CDCl}_{3}\right): \delta=21.8\left(\mathrm{CH}_{3}, 2 \mathrm{C}\right), 42.8\left(\mathrm{CH}_{2}\right)$, $68.4\left(\mathrm{CH}_{2}\right), 72.6(\mathrm{CH}), 75.3(\mathrm{CH}), 160.1(\mathrm{C})$.

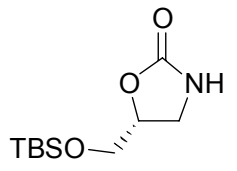

(R)-5-(tert-Butyl-dimethyl-silanyloxymethyl)-oxazolidin-2-one (4g) -

The reaction was carried out following the general procedure at room temperature for $36 \mathrm{~h}$ using $3 \mathrm{~mol} \%$ of $(R, R)$-(salen)Co ${ }^{\mathrm{II}} \mathbf{1}, 6 \mathrm{~mol} \%$ of $p$ nitrobenzoic acid as the additive, $0.5 \mathrm{mmol}$ of urethane $\mathbf{3}$ and $1.05 \mathrm{mmol}$ of racemic epoxide $\mathbf{2 g}$. 
After the cyclization step, the title compound was isolated by column chromatography (DCM/AcOEt $65 / 35$ ) as a white solid in $92 \%$ yield; melting point: $56-58{ }^{\circ} \mathrm{C}$. The ee was determined by HPLC analysis on the corresponding $N$-benzoyl derivative, prepared following the described general procedure, using a Chiralpak AD-H column (80/20 hexane/i-PrOH; flow rate $\left.0.75 \mathrm{~mL} / \mathrm{min} ;=\tau_{\mathrm{S}} 8.4 \mathrm{~min} ; \tau_{\mathrm{R}}=9.0 \mathrm{~min}\right) .[\alpha]_{\mathrm{D}}^{\mathrm{rt}}=-4.7^{\circ}\left(c=1.06, \mathrm{CHCl}_{3}, 99.0 \%\right.$ ee $)$. ESIMS $m / z 232[\mathrm{M}+\mathrm{H}]^{+}, 254[\mathrm{M}+\mathrm{Na}]^{+} .{ }^{1} \mathrm{H}$ NMR $\left(\mathrm{CDCl}_{3}\right): \delta=0.08\left(\mathrm{~s}, 6 \mathrm{H}, 2 \mathrm{CH}_{3}\right), 0.88(\mathrm{~s}, 9 \mathrm{H}, 3$ $\mathrm{CH}_{3}$ ), 3.57 (ABX, J = 8.6, 8.6, 2H), $3.77(\mathrm{ABX}, \mathrm{J}=4.8,11.2,2 \mathrm{H}), 4.63-4.70(\mathrm{~m}, 1 \mathrm{H}), 5.56$ (bs, $1 \mathrm{H}, \mathrm{NH}) ;{ }^{13} \mathrm{C}$ NMR $\left(\mathrm{CDCl}_{3}\right): \delta=-5.5\left(\mathrm{CH}_{3}, 2 \mathrm{C}\right), 18.1(\mathrm{C}), 25.7\left(\mathrm{CH}_{3}, 3 \mathrm{C}\right), 42.1\left(\mathrm{CH}_{2}\right), 63.4$ $\left(\mathrm{CH}_{2}\right), 76.3(\mathrm{CH}), 160.4(\mathrm{C})$.

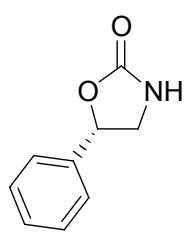

(S)-5-Phenyl-oxazolidin-2-one (4h) $)^{5}$ - The reaction was carried out following the general procedure at room temperature for $40 \mathrm{~h}$ using $4 \mathrm{~mol} \%$ of $(R, R)$ (salen)Co ${ }^{\mathrm{II}} \mathbf{1}, 8 \mathrm{~mol} \%$ of $p$-nitrobenzoic acid as the additive, $0.5 \mathrm{mmol}$ of urethane $\mathbf{3}$ and $1.1 \mathrm{mmol}$ of racemic styrene oxide $\mathbf{2 h}$. After the cyclization step, the title compound was isolated by column chromatography (DCM/AcOEt 8/2) as a white solid in $90 \%$ yield; melting point: $92-93{ }^{\circ} \mathrm{C}$. The ee was determined by HPLC analysis using a Chiralcel OD column $\left(90 / 10\right.$ hexane $/ i-\mathrm{PrOH}$; flow rate $0.75 \mathrm{~mL} / \mathrm{min} ; \tau_{\mathrm{S}}=29.5 \mathrm{~min} ; \tau_{\mathrm{R}}=40.2$ $\min )$. $[\alpha]_{D}^{\mathrm{rt}}=+22.6^{\circ}\left(c=0.93, \mathrm{CHCl}_{3}, 99.2 \%\right.$ ee $)$, lit. $^{4}[\alpha]_{D}^{\mathrm{rt}}=+10.0^{\circ}$, (S)-4h. ESI-MS $m / z 162$ $[\mathrm{M}-\mathrm{H}] \cdot{ }^{1} \mathrm{H}$ NMR $\left(\mathrm{CDCl}_{3}\right): \delta=3.55(\mathrm{ddd}, J=0.8,7.6,8.6,1 \mathrm{H}), 3.98(\mathrm{ddd}, J=0.8,8.6,8.6,1 \mathrm{H})$, $5.50(\mathrm{bs}, 1 \mathrm{H}, \mathrm{NH}), 5.63(\mathrm{t}, J=8.6,1 \mathrm{H}), 7.35-7.43(\mathrm{~m}, 5 \mathrm{H}) ;{ }^{13} \mathrm{C} \mathrm{NMR}\left(\mathrm{CDCl}_{3}\right): \delta=48.3\left(\mathrm{CH}_{2}\right)$, $77.9(\mathrm{CH}), 125.6(\mathrm{CH}, 2 \mathrm{C}), 128.9(\mathrm{CH}, 3 \mathrm{C}), 138.4(\mathrm{C}), 159.9(\mathrm{C})$.

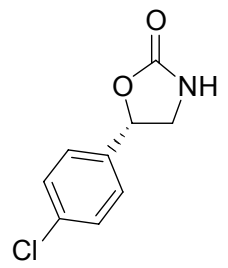

(S)-5-(4-Chloro-phenyl)-oxazolidin-2-one (4i) - The reaction was carried out at room temperature for $60 \mathrm{~h}$ using $5 \mathrm{~mol} \%$ of $(R, R)-(\operatorname{salen}) \mathrm{Co}^{\mathrm{II}} \mathbf{1}, 8 \mathrm{~mol} \%$ of $p$-nitrobenzoic acid as the additive, $0.5 \mathrm{mmol}$ of urethane 3 and $1.1 \mathrm{mmol}$ of racemic epoxide $\mathbf{2 i}$. The cyclization was accomplished by adding THF $(0.1 \mathrm{M})$ and tert-BuOK (2 equiv). After $16 \mathrm{~h}$ stirring, the crude reaction mixture was quenched with $\mathrm{NH}_{4} \mathrm{Cl}$ and diluted with $\mathrm{Et}_{2} \mathrm{O}$. The organic layer was washed with brine and dried over $\mathrm{Na}_{2} \mathrm{SO}_{4}$. After filtration, solvent was removed in vacuo, and the title compound was isolated by column chromatography (DCM/AcOEt 7/3) as a white solid in $98 \%$ yield; melting point: $129-132{ }^{\circ} \mathrm{C}$. The ee was determined by HPLC analysis using a Chiralcel OD column (90/10 hexane $/ i-\mathrm{PrOH}$; flow rate $\left.0.75 \mathrm{~mL} / \mathrm{min} ; \tau_{\mathrm{S}}=35.3 \mathrm{~min} ; \tau_{\mathrm{R}}=40.8 \mathrm{~min}\right) .[\alpha]^{\mathrm{rt}}{ }_{\mathrm{D}}=$ $+34.2^{\circ}\left(c=1.05, \mathrm{CHCl}_{3}, 98.7 \%\right.$ ee $)$. ESI-MS $m / z 196[\mathrm{M}-\mathrm{H}]^{-} .{ }^{1} \mathrm{H}$ NMR $\left(\mathrm{CDCl}_{3}\right): \delta=3.50(\mathrm{ddd}$, $J=0.8,7.6,8.4,1 \mathrm{H}), 3.98(\mathrm{ddd}, J=0.8,8.4,8.4,1 \mathrm{H}), 5.60$ (t, $J=8.4,1 \mathrm{H}), 5.85$ (bs, $1 \mathrm{H}, \mathrm{NH})$, 
7.30-7.40 (m, 4H); ${ }^{13} \mathrm{C}$ NMR $\left(\mathrm{CDCl}_{3}\right): \delta=48.2\left(\mathrm{CH}_{2}\right), 77.1(\mathrm{CH}), 127.0(\mathrm{CH}, 2 \mathrm{C}), 129.1(\mathrm{CH}$, 2C), 134.8 (C), 136.9 (C), 159.7 (C).

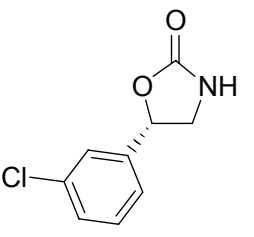

(S)-5-(3-Chloro-phenyl)-oxazolidin-2-one (4j) - The reaction was carried out following the general procedure at room temperature for $60 \mathrm{~h}$ using 5 mol\% of $(R, R)$-(salen) $\mathrm{Co}^{\mathrm{II}} \mathbf{1}, 8 \mathrm{~mol} \%$ of $p$-nitrobenzoic acid as the additive, $0.5 \mathrm{mmol}$ of urethane $\mathbf{3}$ and $1.1 \mathrm{mmol}$ of racemic epoxide $\mathbf{2 j}$. After the cyclization step, the title compound was isolated by column chromatography (DCM/AcOEt 7/3) as a white solid in $97 \%$ yield; melting point: $114-116^{\circ} \mathrm{C}$. The ee was determined by HPLC analysis using a Chiralcel OD column $\left(90 / 10\right.$ hexane $/ i-\mathrm{PrOH}$; flow rate $0.75 \mathrm{~mL} / \mathrm{min} ; \tau_{\mathrm{S}}=32.0$ $\left.\min ; \tau_{\mathrm{R}}=42.1 \mathrm{~min}\right) .[\alpha]^{\mathrm{rt}} \mathrm{D}=+37.2^{\circ}\left(c=1.07, \mathrm{CHCl}_{3}, 96.5 \%\right.$ ee $)$. ESI-MS $m / z 196[\mathrm{M}-\mathrm{H}]^{-} .{ }^{1} \mathrm{H}$ NMR $\left(\mathrm{CDCl}_{3}\right): \delta=3.51(\mathrm{ddd}, J=0.8,7.6,8.4,1 \mathrm{H}), 4.00(\mathrm{ddd}, J=0.8,8.4,8.4,1 \mathrm{H}), 5.60$ (t, $J=$ 8.4, $1 \mathrm{H}), 5.82$ (bs, $1 \mathrm{H}, \mathrm{NH}), 7.25-7.40(\mathrm{~m}, 4 \mathrm{H}) ;{ }^{13} \mathrm{C} \mathrm{NMR}\left(\mathrm{CDCl}_{3}\right): \delta=48.1\left(\mathrm{CH}_{2}\right), 76.9(\mathrm{CH})$, 123.6 (CH), $125.8(\mathrm{CH}), 129.0(\mathrm{CH}), 130.2(\mathrm{CH}), 134.9(\mathrm{C}), 140.5(\mathrm{C}), 159.7(\mathrm{C})$.

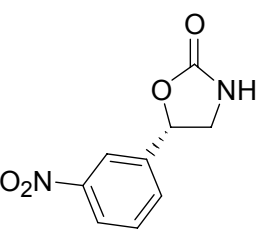

(S)-5-(3-Nitro-phenyl)-oxazolidin-2-one (4k) - The reaction was carried out following the general procedure at room temperature for $60 \mathrm{~h}$ using $5 \mathrm{~mol} \%$ of $(R, R)$-(salen) $\mathrm{Co}^{\mathrm{II}} \mathbf{1}, 8 \mathrm{~mol} \%$ of $p$-nitrobenzoic acid as the additive, $0.5 \mathrm{mmol}$ of urethane $\mathbf{3}$ and $1.1 \mathrm{mmol}$ of racemic epoxide $\mathbf{2 k}$. After the cyclization step, the title compound was isolated by column chromatography $(\mathrm{DCM} / \mathrm{AcOEt} 7 / 3)$ as a white solid in $94 \%$ yield; melting point: $101-103{ }^{\circ} \mathrm{C}$. The ee was determined by HPLC analysis using a Chiralpak OB-H column (70/30 hexane/i-PrOH; flow rate $\left.0.75 \mathrm{~mL} / \mathrm{min} ; \tau_{\mathrm{R}}=45.4 \mathrm{~min} ; \tau_{\mathrm{S}}=52.3 \mathrm{~min}\right) .[\alpha]_{\mathrm{D}}^{\mathrm{rt}}=+15.5^{\circ}\left(c=1.0, \mathrm{CHCl}_{3}, 97.5 \%\right.$ ee $)$.ESI-MS $m / z 207[\mathrm{M}-\mathrm{H}]^{-} .{ }^{1} \mathrm{H}$ NMR $\left(\mathrm{CDCl}_{3}\right): \delta=3.56(\mathrm{ddd}, J=0.8,7.6,8.4,1 \mathrm{H}), 4.10(\mathrm{ddd}, J=0.8,8.4$, 8.4, 1H), 5.43 (bs, $1 \mathrm{H}, \mathrm{NH}), 5.74(\mathrm{t}, J=8.4,1 \mathrm{H}), 7.61-7.66(\mathrm{~m}, 1 \mathrm{H}), 7.75-7.78(\mathrm{~m}, 1 \mathrm{H}), 8.23-$ $8.27(\mathrm{~m}, 2 \mathrm{H}) ;{ }^{13} \mathrm{C}$ NMR $\left(\mathrm{CDCl}_{3}\right): \delta=48.0\left(\mathrm{CH}_{2}\right), 76.4(\mathrm{CH}), 120.7(\mathrm{CH}), 123.9(\mathrm{CH}), 130.2$ $(\mathrm{CH}), 131.5(\mathrm{CH}), 140.6(\mathrm{C}), 148.5(\mathrm{C}), 159.1(\mathrm{C})$. 
Enantioconvergent Strategy for the Total Conversion of Racemic Epoxide into a Single Enantiopure Oxazolidinone.

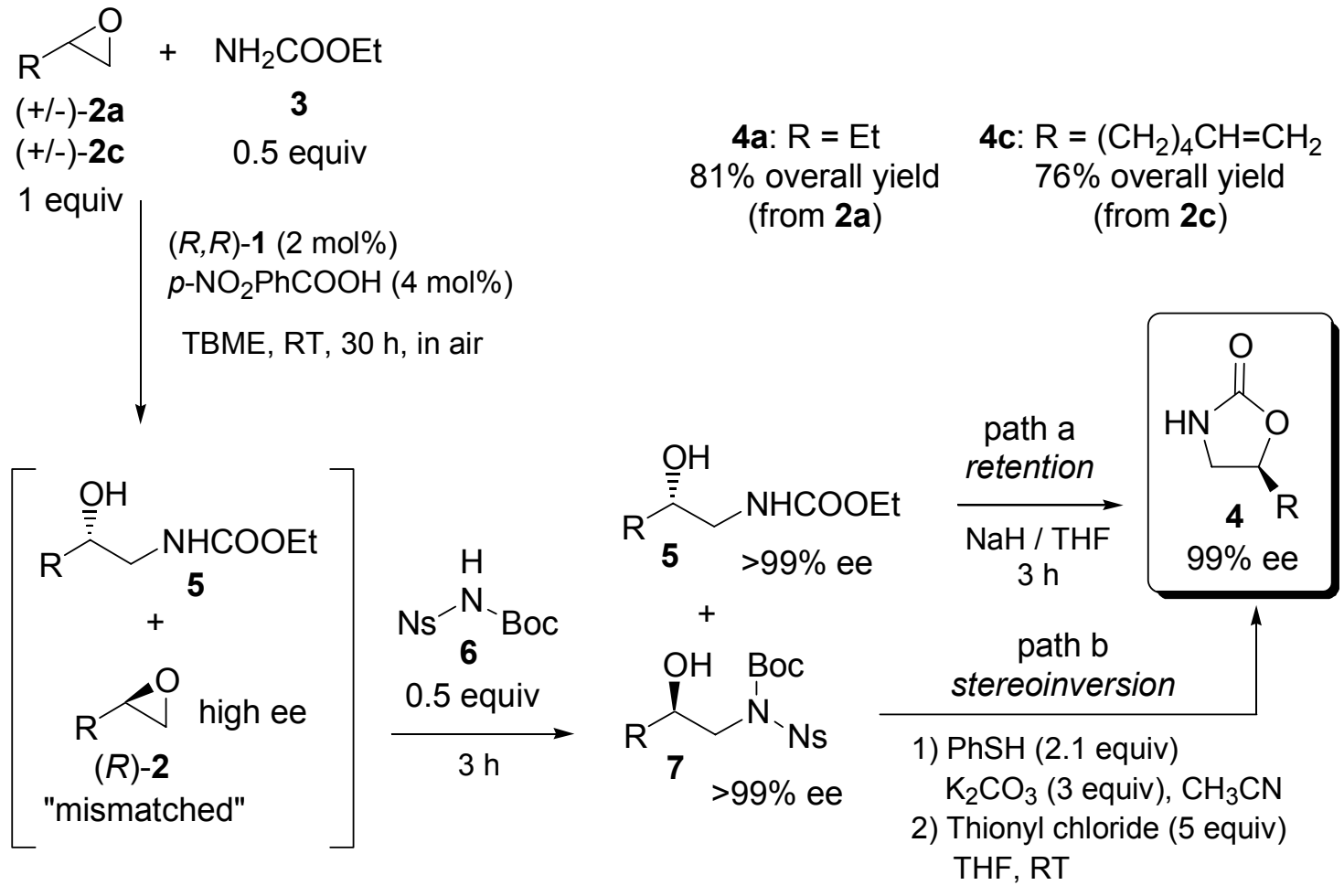

\section{Total conversion of racemic 2a into enantiopure oxazolidinone 4a.}

The reaction was carried out in undistilled solvent without any precautions to exclude water. In an ordinary test tube equipped with a magnetic stirring bar, the (salen)-Co ${ }^{\mathrm{II}}$ complex 1 (24 mg, $0.04 \mathrm{mmol}$ ) was dissolved in $0.15 \mathrm{~mL}$ of TBME. After addition of the oxidizing agent ( $p$ nitrobenzoic acid, $13.3 \mathrm{mg}, 0.08 \mathrm{mmol}$ ), the tube was closed with a rubber stopper and the mixture was stirred at room temperature over which time the color changed from orange-red to dark brown. Then the urethane 3 ( $89 \mathrm{mg}, 1 \mathrm{mmol}$ ) was added. After 5 minutes stirring, epoxide 2a (144 mg, $2 \mathrm{mmol}$ ) was added and stirring was continued at room temperature for $30 \mathrm{~h}$, at which time $0.15 \mathrm{~mL}$ of TBME and $N$-Boc sulfonamide 6 (301 $\mathrm{mg}, 1 \mathrm{mmol})$ were added. After 3 $\mathrm{h}$ the crude reaction mixture was directly charged on the column chromatography and purified (DCM/AcOEt from 95/5 to 8/2) to yield the isolated pseudo-enantiomeric aminols 5a and $7 \mathbf{a}$.<smiles>CCOC(=O)NC[C@H](O)CC</smiles>

((S)-2-Hydroxy-butyl)-carbamic acid ethyl ester (5a) - The title compound was isolated by column chromatography (DCM/AcOEt 8/2, $\mathrm{R}_{f}$ $=0.2)$ in $84 \%$ yield as a colourless liquid. $[\alpha]^{\mathrm{tt}}=+20.6^{\circ}\left(c=1.1, \mathrm{CHCl}_{3}\right)$. ESI-MS $m / z 160[\mathrm{M}-$ $\mathrm{H}^{-}, 184[\mathrm{M}+\mathrm{Na}]^{+} .{ }^{1} \mathrm{H}$ NMR $\left(\mathrm{CDCl}_{3}\right): \delta=0.95(\mathrm{t}, J=7.2,3 \mathrm{H}), 1.23(\mathrm{t}, J=7.0,3 \mathrm{H}), 1.41-1.53$ (m, 2H), 2.57 (bs, 1H, OH), 3.00-3.10 (m, 1H), 3.29-3.39 (m, 1H), 3.55-3.65 (m, 1H), 4.11 (q, $J$ 
$=7.2,2 \mathrm{H}), 5.17(\mathrm{bs}, 1 \mathrm{H}, \mathrm{NH}) ;{ }^{13} \mathrm{C} \mathrm{NMR}\left(\mathrm{CDCl}_{3}\right): \delta=9.7\left(\mathrm{CH}_{3}\right), 14.5\left(\mathrm{CH}_{3}\right), 27.5\left(\mathrm{CH}_{2}\right), 46.4$ $\left(\mathrm{CH}_{2}\right), 60.8\left(\mathrm{CH}_{2}\right), 72.5(\mathrm{CH}), 157.4(\mathrm{C})$.

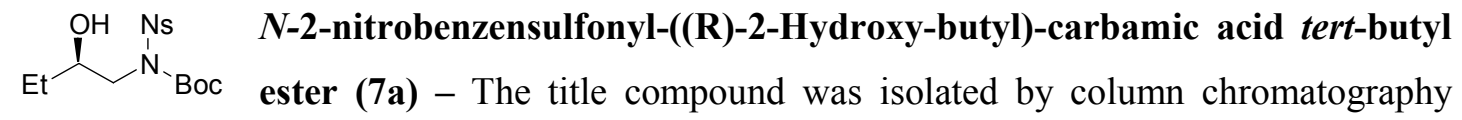
(DCM/AcOEt $8 / 2, \mathrm{R}_{f}=0.6$ ) in $98 \%$ yield as a white solid, melting point: $81-83{ }^{\circ} \mathrm{C}$. The ee was determined by HPLC analysis using a Chiralpak AD-H column $(8 / 2$ hexane/i-PrOH; flow rate $\left.0.75 \mathrm{~mL} / \mathrm{min} ; \tau_{\mathrm{R}}=26.2 \mathrm{~min} ; \tau_{\mathrm{S}}=35.5 \mathrm{~min}\right) .[\alpha]_{\mathrm{D}}^{\mathrm{rt}}=+26.6^{\circ}\left(c=2.0, \mathrm{CHCl}_{3}, 99.5 \%\right.$ ee $)$. ESI-MS $m / z 373[\mathrm{M}-\mathrm{H}]^{-} .{ }^{1} \mathrm{H}$ NMR $\left(\mathrm{CDCl}_{3}\right): 1.03(\mathrm{t}, J=7.0,3 \mathrm{H}), 1.34(\mathrm{~s}, 9 \mathrm{H}), 1.48-1.64(\mathrm{~m}, 2 \mathrm{H}), 2.00$ (d, $J=6.0, \mathrm{OH}), 3.79-3.89(\mathrm{~m}, 3 \mathrm{H}), 7.73-7.79(\mathrm{~m}, 3 \mathrm{H}), 8.34-8.38(\mathrm{~m}, 1 \mathrm{H}) ;{ }^{13} \mathrm{C} \mathrm{NMR}\left(\mathrm{CDCl}_{3}\right): \delta$ $=9.8\left(\mathrm{CH}_{3}\right), 27.8\left(\mathrm{CH}_{3}, 3 \mathrm{C}\right), 27.9\left(\mathrm{CH}_{2}\right), 53.1\left(\mathrm{CH}_{2}\right), 72.1(\mathrm{CH}), 85.5(\mathrm{C}), 124.4(\mathrm{CH}), 131.8$ (CH), $133.1(\mathrm{CH}), 133.6(\mathrm{C}), 134.1(\mathrm{CH}), 147.6(\mathrm{C}), 150.8(\mathrm{C})$.

Cylization of 5 a to Oxazolidinone 4 a with Retention of Configuration: Pure amino alcohol 5 a (136 mg, $0.84 \mathrm{mmol})$ was dissolved in THF $(8.5 \mathrm{~mL})$ and NaH (40 mg, $1.7 \mathrm{mmol})$ was added at room temperature. After being stirred for 3 hours, the mixture was diluted with $\mathrm{Et}_{2} \mathrm{O}$ and the reaction was quenched with some drops of methanol. The mixture was washed with $\mathrm{NH}_{4} \mathrm{Cl}$, extracted with $\mathrm{Et}_{2} \mathrm{O}$ and dried over anhydrous $\mathrm{MgSO}_{4}$. Evaporation of the solvent affords pure oxazolidinone $4 \mathbf{a}$ in quantitative yield $(96.5 \mathrm{mg}$ ) and in enantiopure form (ee $>99 \%$ ).

Cyclization of 7 a to Oxazolidinone 4 a with Inversion of Configuration (one-pot reaction sequence):

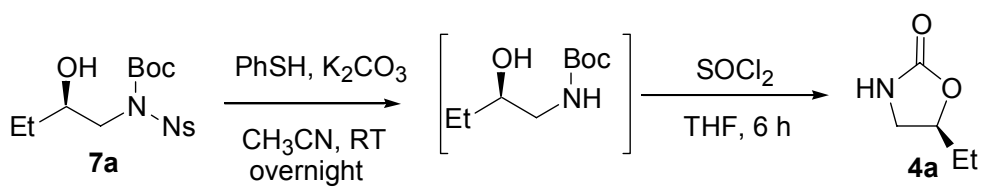

1) Deprotection of the Nosyl group: $:^{9}$ pure amino alcohol 7a (366 mg, $\left.0.98 \mathrm{mmol}\right)$ was dissolved in $\mathrm{CH}_{3} \mathrm{CN}(10 \mathrm{~mL})$, followed by the addition of 1.1 equiv of thiophenol (110 $\mu \mathrm{l}, 1.08 \mathrm{mmol})$ and 2 equiv of $\mathrm{K}_{2} \mathrm{CO}_{3}(270.5 \mathrm{mg}, 1.96 \mathrm{mmol})$. After being stirred for 1 hour at room temperature, 1 equiv of $\mathrm{K}_{2} \mathrm{CO}_{3}(135.2 \mathrm{mg}, 0.98 \mathrm{mmol})$ and 1 equiv of thiophenol ( $108 \mu \mathrm{l}, 0.98 \mathrm{mmol})$ were added. After being stirred overnight, the end of the deprotection step was monitored by TLC analysis.

2) Subsequent One-pot Cyclization with Stereoinversion: ${ }^{10}$ The stereoinversion was achieved by directly adding THF $(10 \mathrm{~mL})$ and 5 equiv of $\mathrm{SOCl}_{2}(356 \mu \mathrm{l}, 4.9 \mathrm{mmol})$.

\footnotetext{
${ }^{9}$ Fukuyama, T.; Cheung, M.; Jow, C.-K.; Hidai Y.; Kan, T. Tetrahedron Lett. 1997,38, 5831.

${ }^{10}$ Ghosh, A. K.; Shin, D.; Mathivanan, P. Chem. Commun. 1999, 1025.
} 
After being stirred at room temperature for 6 hours, the mixture was diluted with AcOEt and quenched with $10 \mathrm{~mL}$ of brine. After extraction of the aqueous layer with AcOEt, the organic layers were collected and dried over anhydrous $\mathrm{MgSO}_{4}$. The title compound 4a was isolated by column chromatography (DCM/AcOEt 6/4, $\mathrm{R}_{f}=0.3$ ) in enantiomerically pure form (ee>99\%) and in $79 \%$ yield $(89 \mathrm{mg}$ ) as a white solid.

Both the cyclization steps afforded the same enantiomer of the oxazolidinone $4 \mathbf{a}$ in $81 \%$ overall yield (186 mg) starting from the racemic 1,2-epoxy butane 2a and in $99.2 \%$ ee.

\section{Total conversion of racemic $2 c$ into enantiopure oxazolidinone $4 c$.}

The reaction was carried out in undistilled solvent without any precautions to exclude water. In an ordinary test tube equipped with a magnetic stirring bar, the (salen)-Co ${ }^{\mathrm{II}}$ complex 1 (24 mg, $0.04 \mathrm{mmol}$ ) was dissolved in $0.15 \mathrm{~mL}$ of TBME. After addition of the oxidizing agent ( $p$ nitrobenzoic acid, $13.3 \mathrm{mg}, 0.08 \mathrm{mmol}$ ), the tube was closed with a rubber stopper and the mixture was stirred at room temperature over which time the color changed from orange-red to dark brown. Then the urethane $\mathbf{3}(89 \mathrm{mg}, 1 \mathrm{mmol})$ was added. After 5 minutes stirring, epoxide 2c (252 mg, $2 \mathrm{mmol}$ ) was added and stirring was continued at room temperature for $30 \mathrm{~h}$, at which time $0.2 \mathrm{~mL}$ of TBME and $N$-Boc sulfonamide 6 (302 $\mathrm{mg}, 1 \mathrm{mmol})$ were added. After 3 $\mathrm{h}$ the crude reaction mixture was directly charged on the column chromatography and purified (DCM/AcOEt from 99/1 to 7/3) to yield the isolated pseudo-enantiomeric aminols $\mathbf{5 c}$ and $\mathbf{7 c}$.

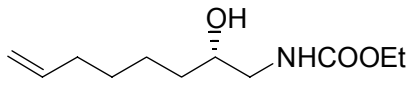

((S)-2-Hydroxy-oct-7-enyl)-carbamic acid ethyl ester (5c) -

The title compound was isolated by column chromatography (DCM/AcOEt 8/2, $\left.\mathrm{R}_{f}=0.25\right)$ in $94 \%$ yield $(202 \mathrm{mg}, 0.94 \mathrm{mmol}$ ) as a white solid, melting point $=38-40{ }^{\circ} \mathrm{C} .[\alpha]^{\mathrm{rt}}=+11.3^{\circ}\left(c=1.1, \mathrm{CHCl}_{3}\right)$. ESI-MS $m / z 214[\mathrm{M}-\mathrm{H}]^{-}, 238[\mathrm{M}+\mathrm{Na}]^{+} .{ }^{1} \mathrm{H} \mathrm{NMR}$ $\left(\mathrm{CDCl}_{3}\right): \delta=1.25(\mathrm{t}, J=7.2,3 \mathrm{H}), 1.35-1.50(\mathrm{~m}, 6 \mathrm{H}), 2.03-2.10(\mathrm{~m}, 2 \mathrm{H}), 2.17$ (bs, $\left.1 \mathrm{H}, \mathrm{OH}\right)$, 3.01-3.09 (m, 1H), 3.32-3.40 (m, 1H), 3.66-3.74 (m, 1H), 4.12 (q, $J=7.2,2 \mathrm{H}), 4.92-5.07(\mathrm{~m}, 3$ $\mathrm{H}, 1 \mathrm{NH}), 5.74-5.85(\mathrm{~m}, 1 \mathrm{H}) ;{ }^{13} \mathrm{C} \mathrm{NMR}\left(\mathrm{CDCl}_{3}\right): \delta=14.5\left(\mathrm{CH}_{3}\right), 24.9\left(\mathrm{CH}_{2}\right), 28.7\left(\mathrm{CH}_{2}\right), 33.5$ $\left(\mathrm{CH}_{2}\right), 34.4\left(\mathrm{CH}_{2}\right), 46.8\left(\mathrm{CH}_{2}\right), 60.9\left(\mathrm{CH}_{2}\right), 71.1(\mathrm{CH}), 114.4\left(\mathrm{CH}_{2}\right), 136.6(\mathrm{CH}), 157.4(\mathrm{C})$.

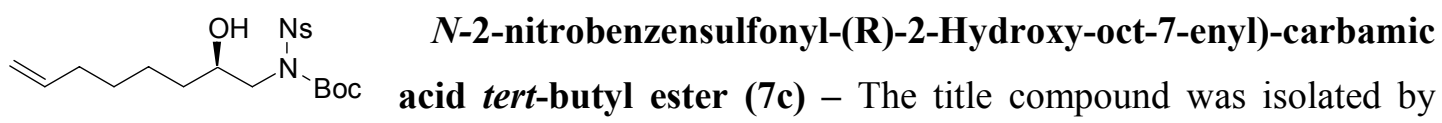
column chromatography (DCM/AcOEt $\left.8 / 2, \mathrm{R}_{f}=0.7\right)$ in $98 \%$ yield $(419 \mathrm{mg}, 0.98 \mathrm{mmol})$ as a white solid, melting point: $103-105{ }^{\circ} \mathrm{C}$. The ee was determined by HPLC analysis using a Chiralpak AD-H column $\left(8 / 2\right.$ hexane $/ i-\mathrm{PrOH}$; flow rate $0.75 \mathrm{~mL} / \mathrm{min} ; \tau_{\mathrm{R}}=22.1 \mathrm{~min} ; \tau_{\mathrm{S}}=31.8$ 
$\min ) .[\alpha]^{\mathrm{rt}}{ }_{\mathrm{D}}=+27.2^{\circ}\left(c=2.05, \mathrm{CHCl}_{3}, 99.7 \%\right.$ ee $)$. ESI-MS $m / z 427[\mathrm{M}-\mathrm{H}]^{-1} .{ }^{1} \mathrm{H}$ NMR $\left(\mathrm{CDCl}_{3}\right): \delta$ $=1.34(\mathrm{~s}, 9 \mathrm{H}), 1.40-1.58(\mathrm{~m}, 6 \mathrm{H}), 2.0(\mathrm{~d}, J=6.4,1 \mathrm{H}, \mathrm{OH}), 2.05-2.11(\mathrm{~m}, 2 \mathrm{H}), 3.76-3.86(\mathrm{~m}$, $2 \mathrm{H}), 3.87-3.95(\mathrm{~m}, 1 \mathrm{H}), 4.92-5.04(\mathrm{~m}, 2 \mathrm{H}), 5.76-5.86(\mathrm{~m}, 1 \mathrm{H}), 7.73-7.77(\mathrm{~m}, 3 \mathrm{H}), 8.34-8.38(\mathrm{~m}$, $1 \mathrm{H}) ;{ }^{13} \mathrm{C}$ NMR $\left(\mathrm{CDCl}_{3}\right): \delta=24.7\left(\mathrm{CH}_{2}\right), 26.6\left(\mathrm{CH}_{3}, 3 \mathrm{C}\right), 28.6\left(\mathrm{CH}_{2}\right), 33.5\left(\mathrm{CH}_{2}\right), 34.6\left(\mathrm{CH}_{2}\right)$, $53.2\left(\mathrm{CH}_{2}\right), 70.5(\mathrm{CH}), 85.4(\mathrm{C}), 114.3\left(\mathrm{CH}_{2}\right), 124.3(\mathrm{CH}), 131.7(\mathrm{CH}), 132.8(\mathrm{CH}), 133.3$ (C), $134.6(\mathrm{CH}), 138.6(\mathrm{CH}), 147.4(\mathrm{C}), 150.6(\mathrm{C})$.

Cylization of 5c to Oxazolidinone 4c with Retention of Configuration: Pure amino alcohol 5c (202 mg, $0.94 \mathrm{mmol})$ was dissolved in THF (10 mL) and NaH (46 mg, $1.9 \mathrm{mmol})$ was added at room temperature. After being stirred for 3 hours, the mixture was diluted with $\mathrm{Et}_{2} \mathrm{O}$ and the reaction was quenched with some drops of methanol. The mixture was washed with $\mathrm{NH}_{4} \mathrm{Cl}$, extracted with $\mathrm{Et}_{2} \mathrm{O}$ and dried over anhydrous $\mathrm{MgSO}_{4}$. Evaporation of the solvent affords pure oxazolidinone $4 \mathbf{c}$ in $98 \%$ yield $(155.5 \mathrm{mg})$ and in enantiopure form $(\mathrm{ee}=98.9 \%)$.

\section{Cyclization of $7 c$ to Oxazolidinone 4c with Inversion of Configuration:}

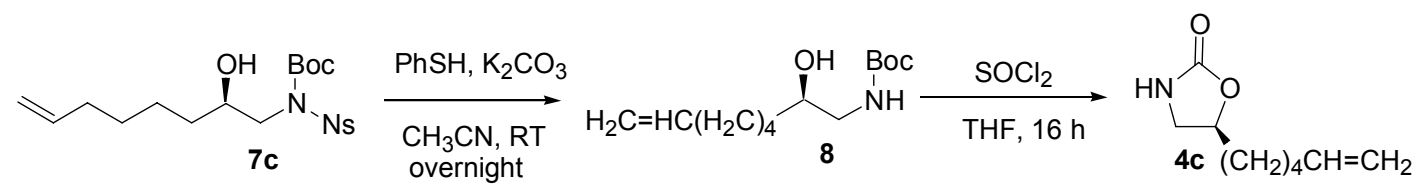

The one-pot reaction sequence afforded the oxazolidinone $\mathbf{4} \mathbf{c}$ with inversion of configuration in $98.0 \%$ ee and in $61 \%$ yield $(101 \mathrm{mg})$. In this case, the isolation of the deprotected aminol $\mathbf{8}$ followed by subsequent cyclization proved to be a better protocol.

1) Deprotection of the Nosyl group: ${ }^{9}$ Pure amino alcohol 7c (419 mg, $\left.0.98 \mathrm{mmol}\right)$ was dissolved in $\mathrm{CH}_{3} \mathrm{CN}(10 \mathrm{~mL})$, followed by the addition of 1.1 equiv of thiophenol (110 $\mu 1,1.08 \mathrm{mmol})$ and 2 equiv of $\mathrm{K}_{2} \mathrm{CO}_{3}(270.5 \mathrm{mg}, 1.96 \mathrm{mmol})$. After being stirred for 1 hour at room temperature, 1 equiv of $\mathrm{K}_{2} \mathrm{CO}_{3}(135.2 \mathrm{mg}, 0.98 \mathrm{mmol})$ and 1 equiv of thiophenol (108 $\mu \mathrm{l}, 0.98 \mathrm{mmol})$ were added. After being stirred overnight, the end of the deprotection step was monitored by TLC analysis. The mixture was quenched with brine, extracted with AcOEt and dried over anhydrous $\mathrm{MgSO}_{4}$. Evaporation of the solvent followed by flash chromatography (DCM/AcOEt 8/2) affords pure aminol $\mathbf{8}$ in $71 \%$ yield (169 mg).

2) Subsequent One-pot Cyclization with Stereoinversion: ${ }^{10}$ Pure aminol 8 was dissolved in THF $(10 \mathrm{~mL})$ and 5 equiv of $\mathrm{SOCl}_{2}(356 \mu \mathrm{l}, 4.9 \mathrm{mmol})$ was added. After being stirred at room temperature for 16 hours, the mixture was diluted with AcOEt and quenched with $10 \mathrm{~mL}$ of brine. After extraction with AcOEt, the organic layers were collected and 
dried over anhydrous $\mathrm{MgSO}_{4}$. The title compound was isolated by column chromatography (DCM/AcOEt 8/2) in quantitative yield $(118 \mathrm{mg})$ and 99.9\% ee.

Both the cyclization steps afforded the same enantiomer of the oxazolidinone $4 \mathbf{c}$ in $82 \%$ overall yield ( $273.5 \mathrm{mg}$ ) starting from racemic 1,2-epoxy-7-octene $\mathbf{2 c}$ and in $>99 \%$ ee. 


\section{HPLC and GC Traces}

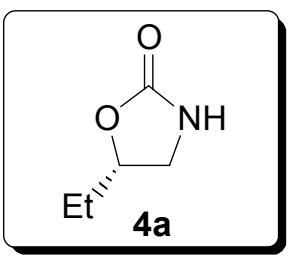

Chiral GC: RT-BetaDEX-sm, isotherm $150{ }^{\circ} \mathrm{C}$
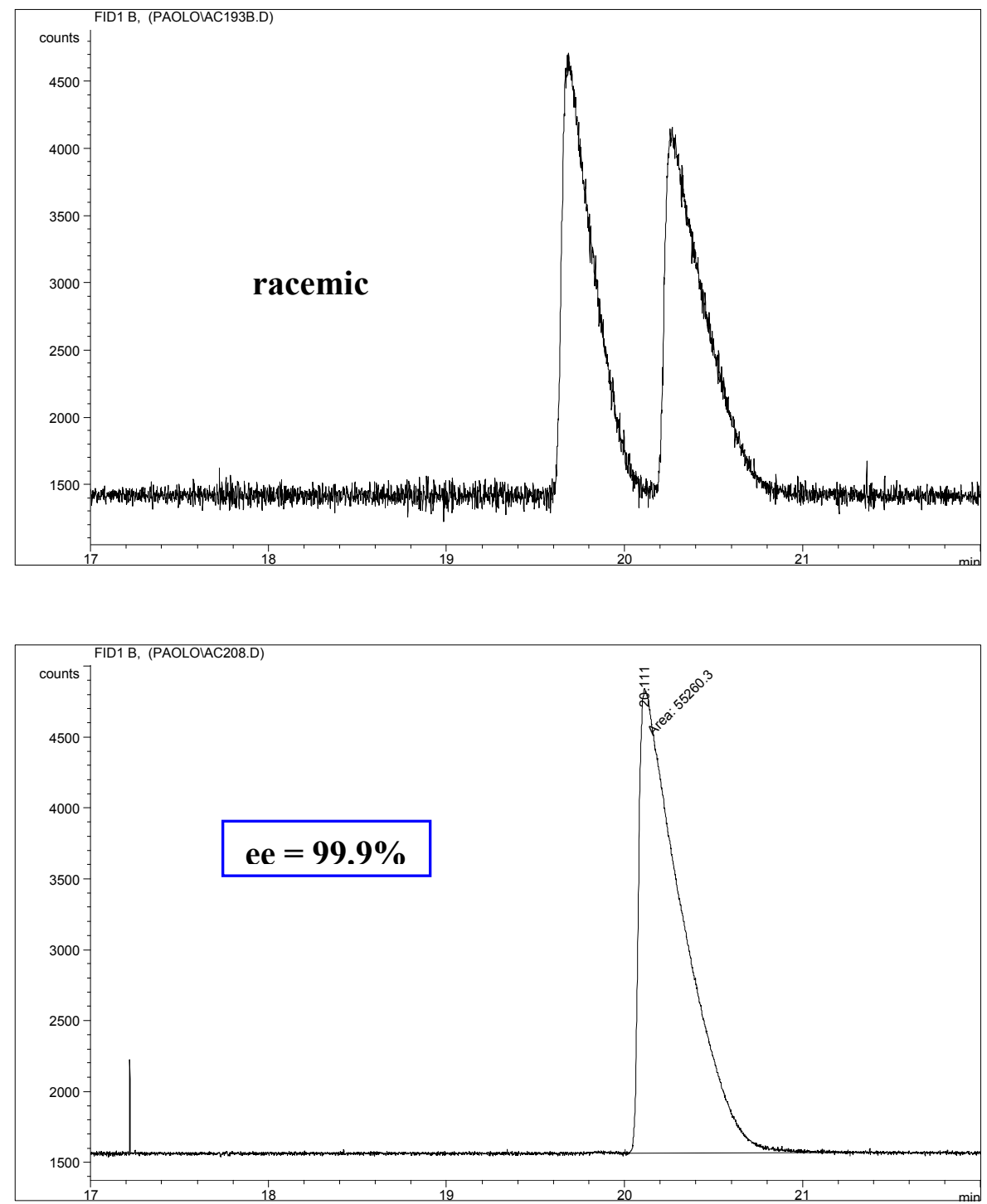

Retention Times

Area

Area Percent

\begin{tabular}{ccc}
\hline \hline 19.686 & nd & 0 \\
20.111 & $5.52603 \cdot 10^{4}$ & 99.99 \\
\hline Totals & $5.52603 \cdot 10^{4}$ &
\end{tabular}




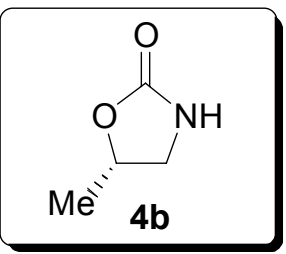

Chiral GC: RT-BetaDEX-sm, isotherm $145^{\circ} \mathrm{C}$
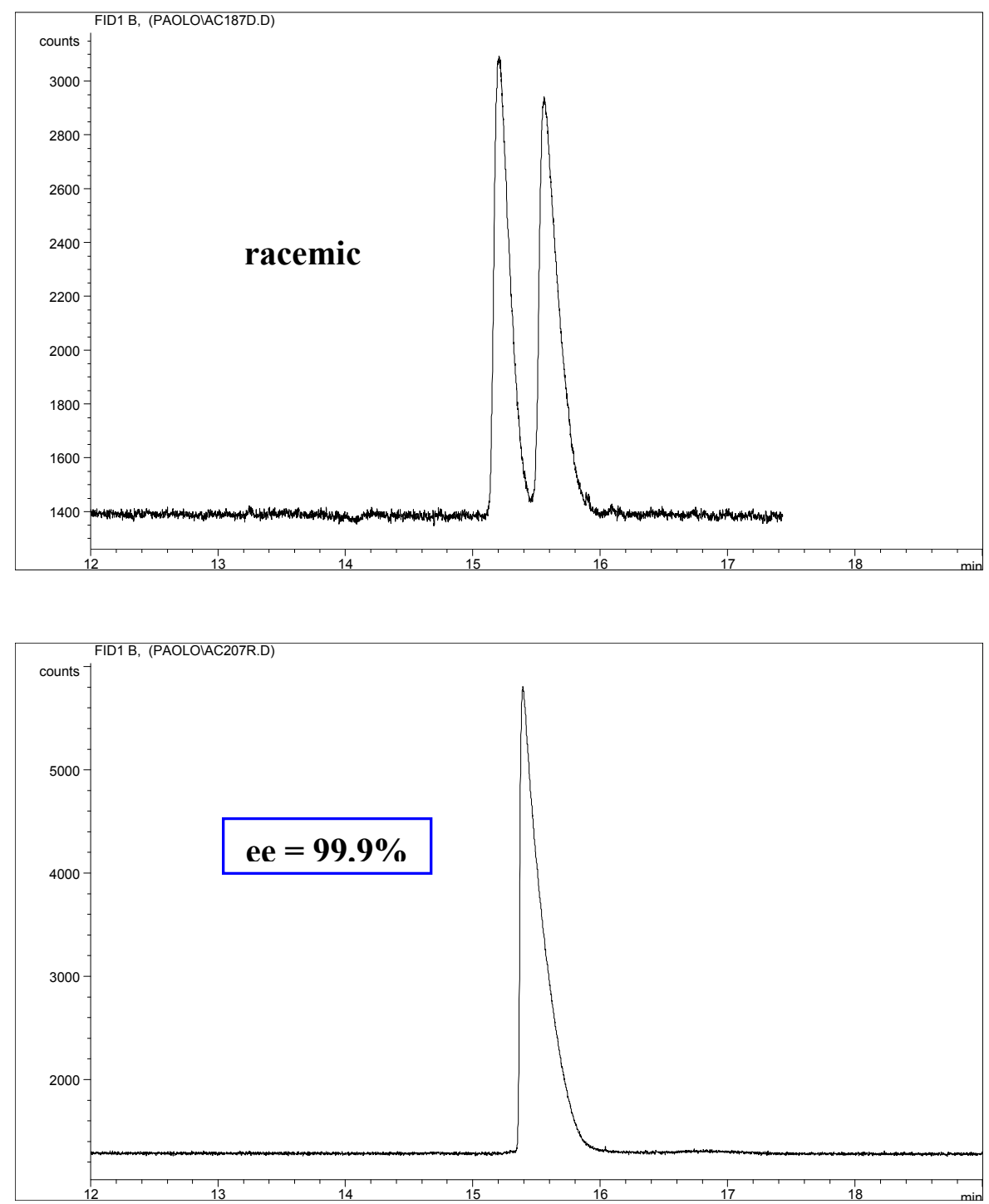

\begin{tabular}{ccc} 
Retention Times & Area & Area Percent \\
\hline \hline 15.207 & nd & 0 \\
15.559 & $5.53331 \cdot 10^{4}$ & 99.99 \\
\hline Totals & $5.53331 \cdot 10^{4}$ &
\end{tabular}




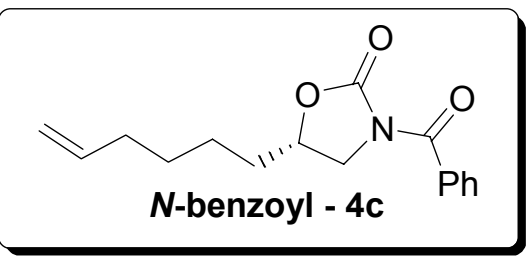

HPLC: CHIRALPAK AD-H 90/10 hexane/iso-propanol, $0.75 \mathrm{~mL} / \mathrm{min}$

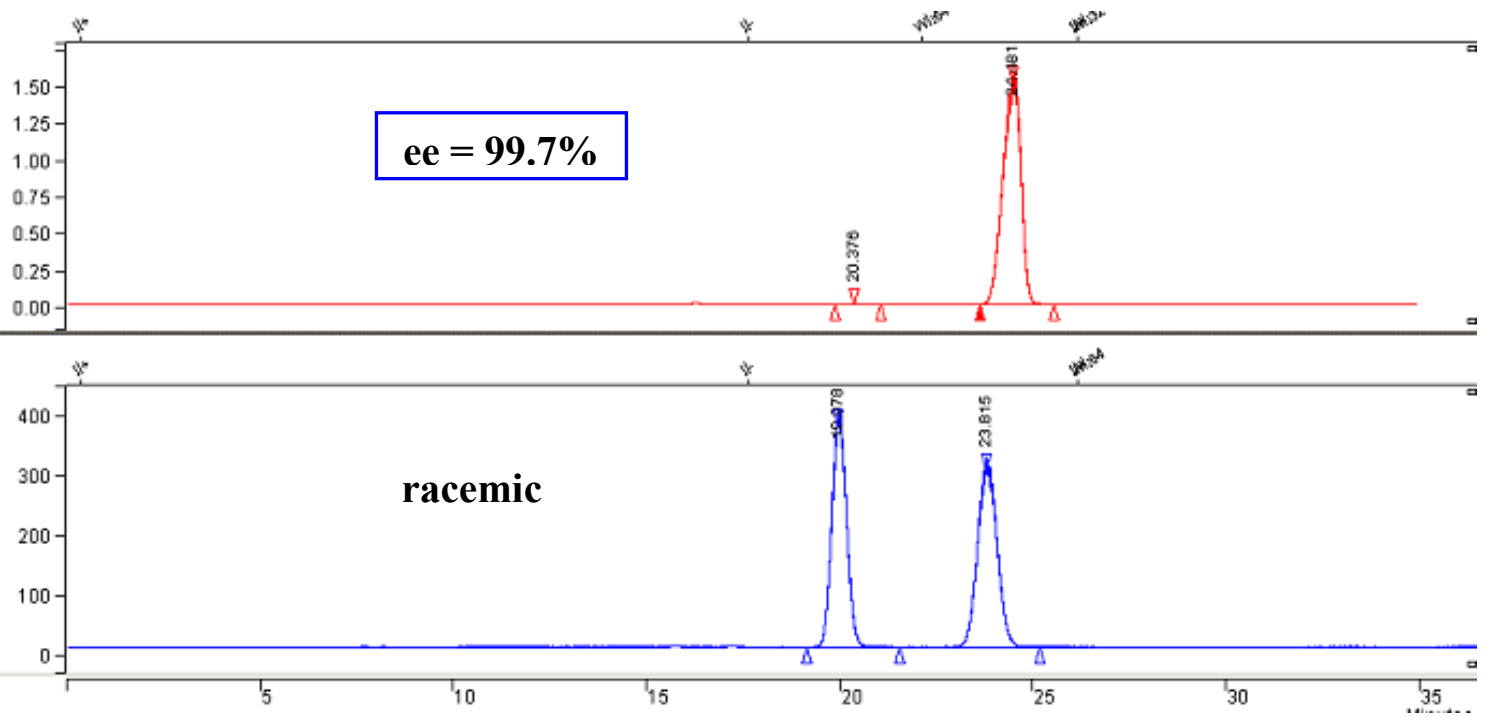

\begin{tabular}{ccc} 
Retention Times & Area & Area Percent \\
\hline \hline 20.376 & 59187 & 0.1192 \\
24.481 & 49604768 & 99.8808 \\
\hline Totals & 49663955 &
\end{tabular}




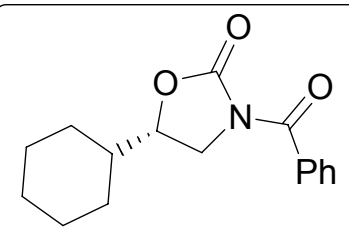

$N$-benzoyl - 4d

HPLC: CHIRALPAK AD-H 85/15 hexane / iso-propanol, $0.75 \mathrm{~mL} / \mathrm{min}$
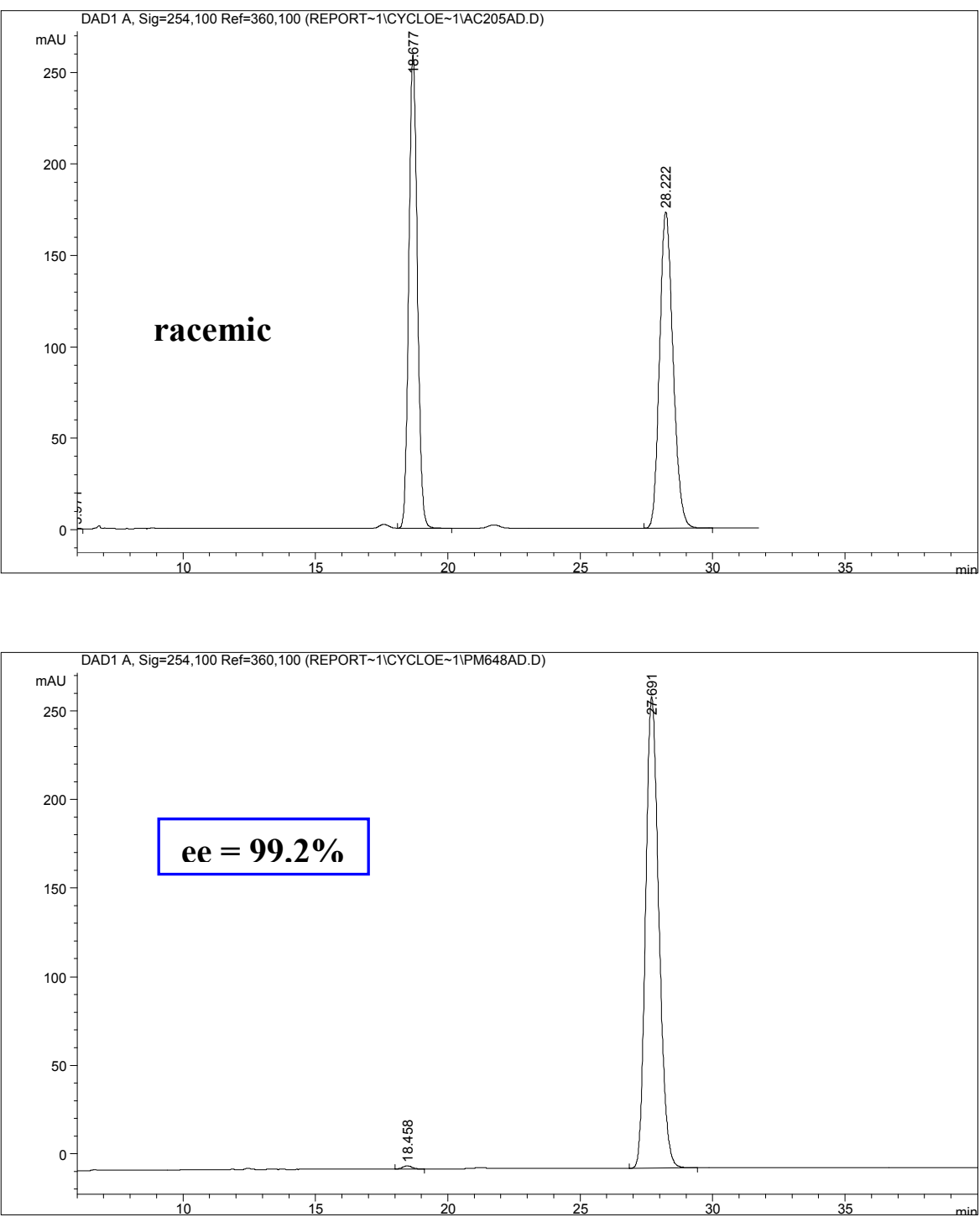

Retention Times

Area

Area Percent

18.458

38.02682

0.4106

27.691

9222.43457

99.5894

Totals

9260.46139 


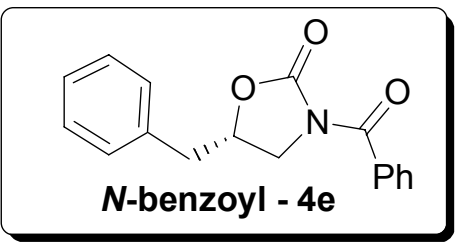

HPLC: CHIRALPAK AS-H 80/20 hexane / iso-propanol, $0.75 \mathrm{~mL}$

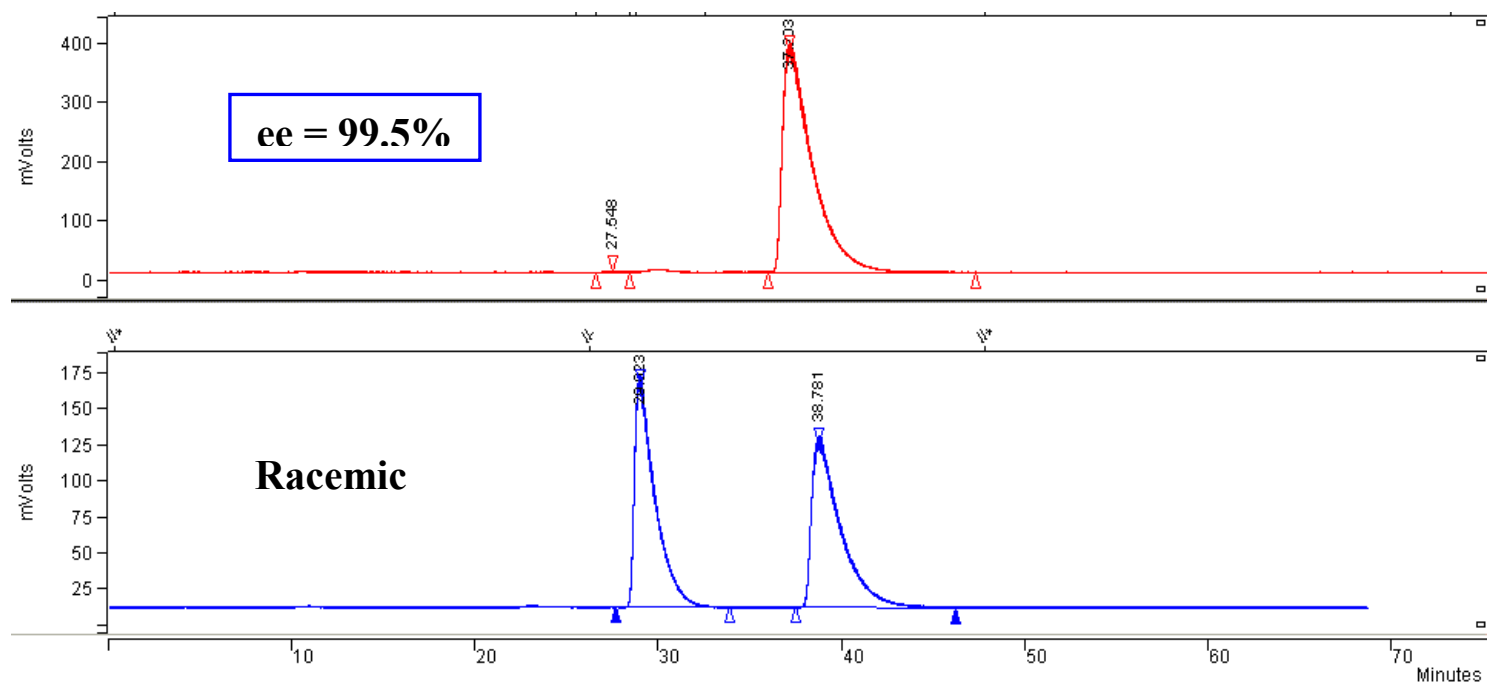

\begin{tabular}{ccc} 
Retention Times & Area & Area Percent \\
\hline \hline 27.548 & 115780 & 0.2694 \\
37.203 & 42862712 & 99.7306 \\
\hline
\end{tabular}

Totals

42978492 


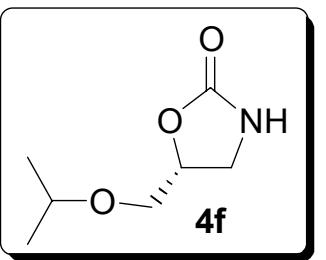

Chiral GC: RT-BetaDEX-sm, isotherm $170{ }^{\circ} \mathrm{C}$
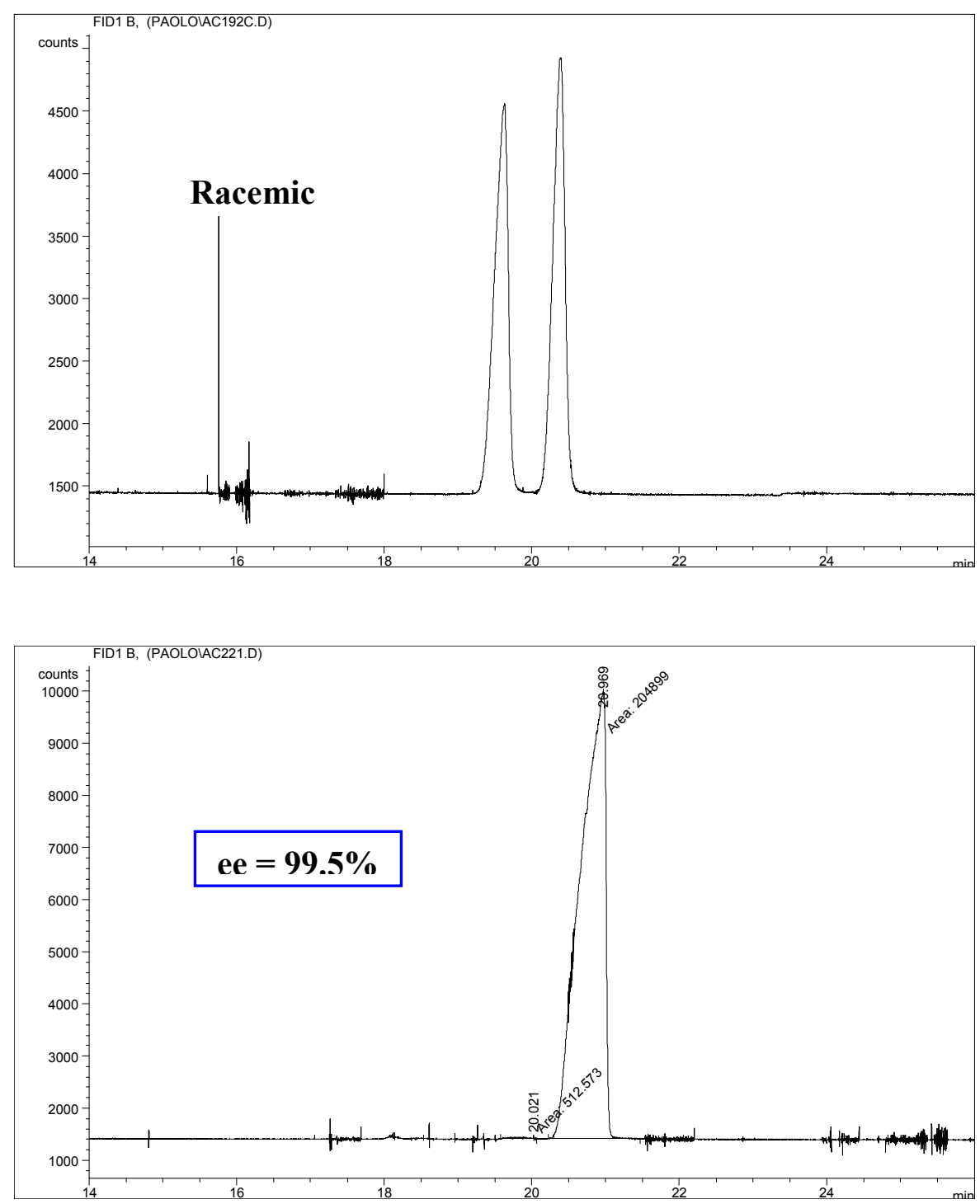

Retention Times

Area

Area Percent

\begin{tabular}{ccc}
\hline \hline 20.021 & 512.57257 & 0.24953 \\
20.969 & $2.04899 \cdot 10^{5}$ & 99.75047 \\
\hline Totals & $2.05412 \cdot 10^{5}$ & \\
\hline
\end{tabular}




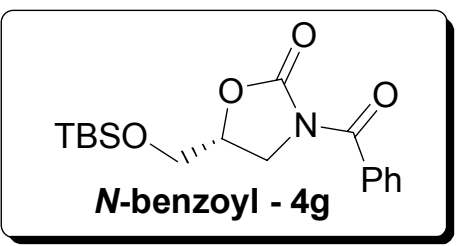

HPLC: CHIRALPAK AD-H 80/20 hexane / iso-propanol, $0.75 \mathrm{~mL}$
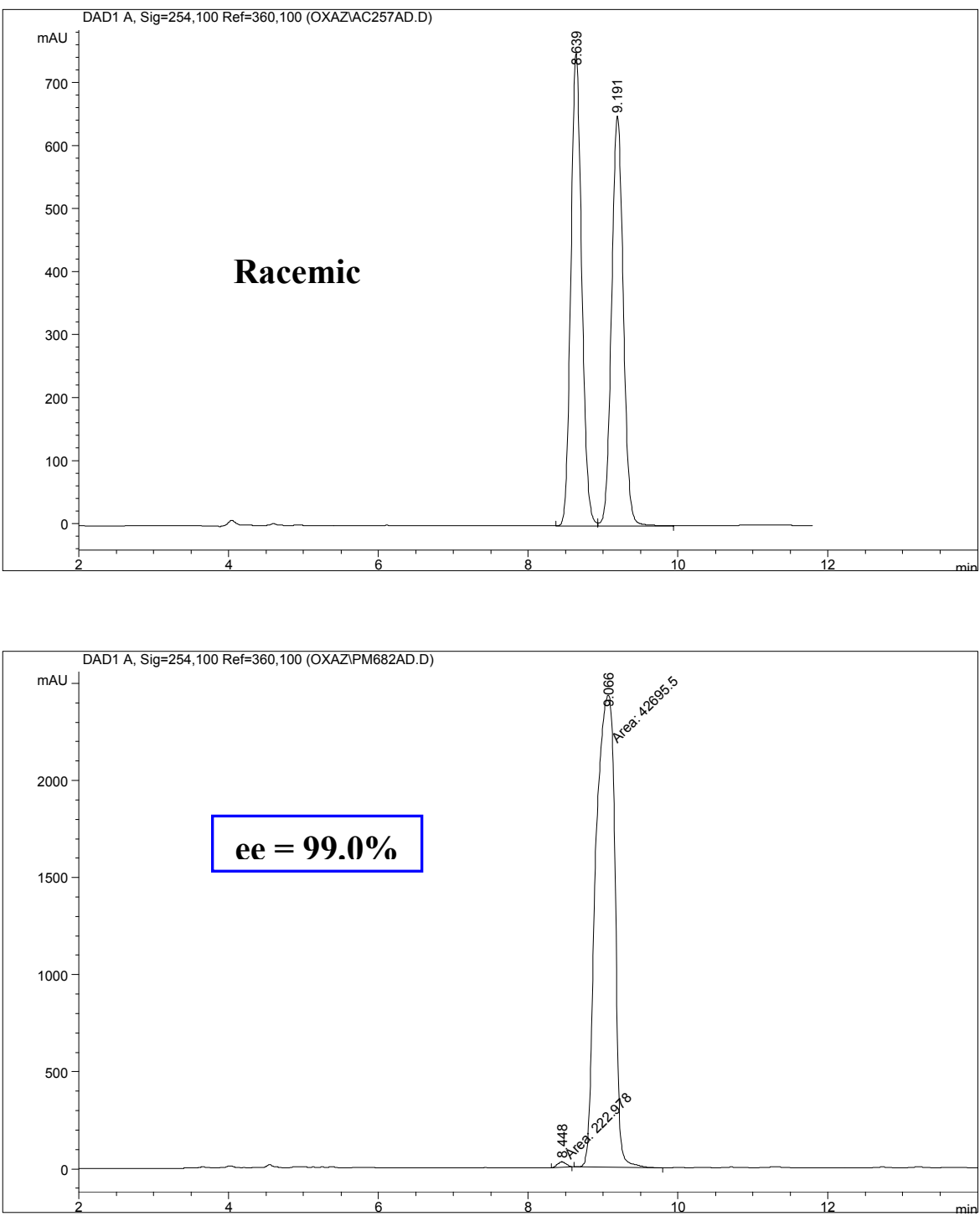

\begin{tabular}{ccc} 
Retention Times & Area & Area Percent \\
\hline \hline 8.448 & 222.97833 & 0.5195 \\
9.066 & $4.26955 \cdot 10^{4}$ & 99.4805 \\
\hline Totals & $4.29184 \cdot 10^{4}$ & \\
\hline
\end{tabular}




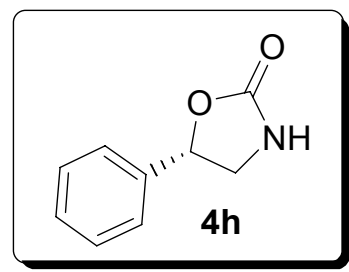

HPLC: CHIRALCEL OD 90/10 hexane / iso-propanol, $0.75 \mathrm{~mL}$

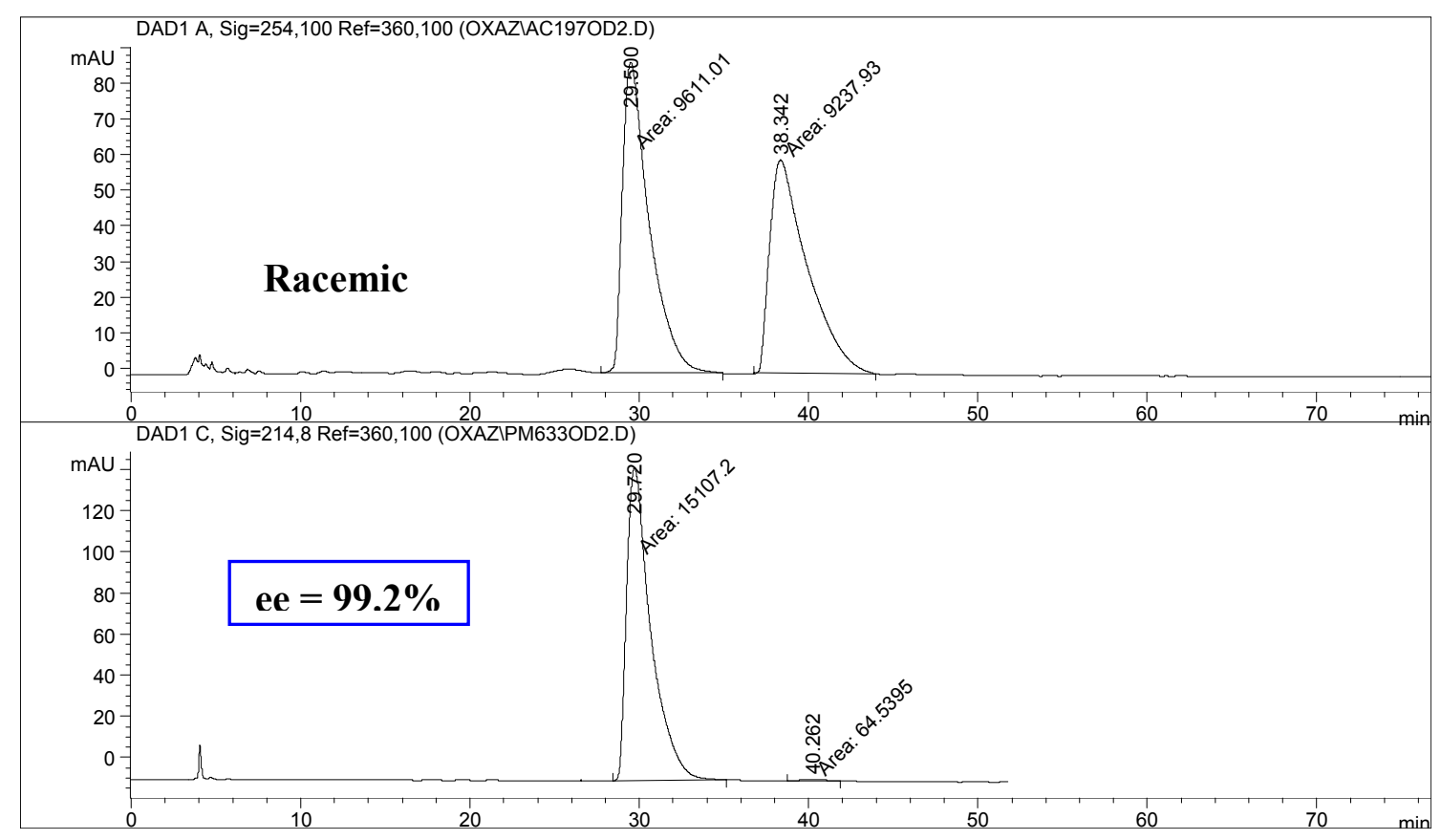

\begin{tabular}{ccc} 
Retention Times & Area & Area Percent \\
\hline \hline 29.720 & $1.51072 \cdot 10^{4}$ & 99.5746 \\
40.262 & 64.53952 & 0.4254 \\
\hline Totals & $1.51717 \cdot 10^{4}$ &
\end{tabular}




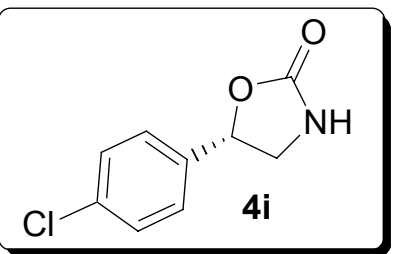

HPLC: CHIRALCEL OD 90/10 hexane / iso-propanol, $0.75 \mathrm{~mL}$

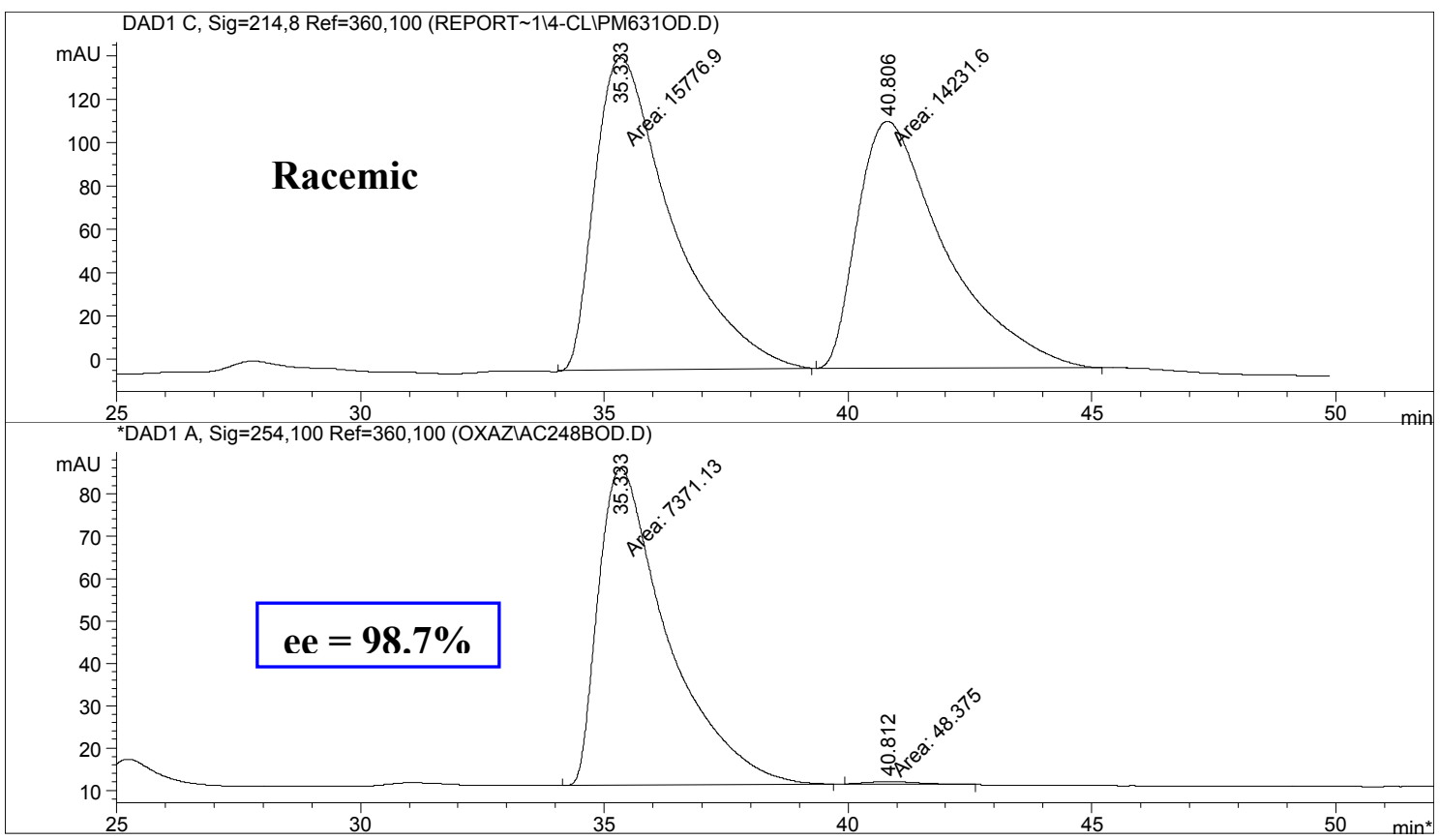

\begin{tabular}{ccc} 
Retention Times & Area & Area Percent \\
\hline \hline 35.333 & 7371.13086 & 99.3480 \\
40.812 & 48.37500 & 0.6520 \\
\hline Totals & 7419.50586 &
\end{tabular}




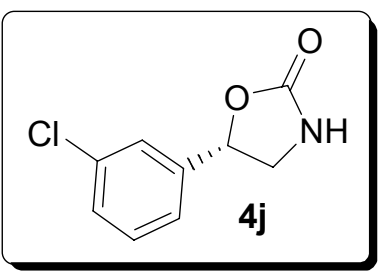

HPLC: CHIRALCEL OD 90/10 hexane / iso-propanol, $0.75 \mathrm{~mL}$
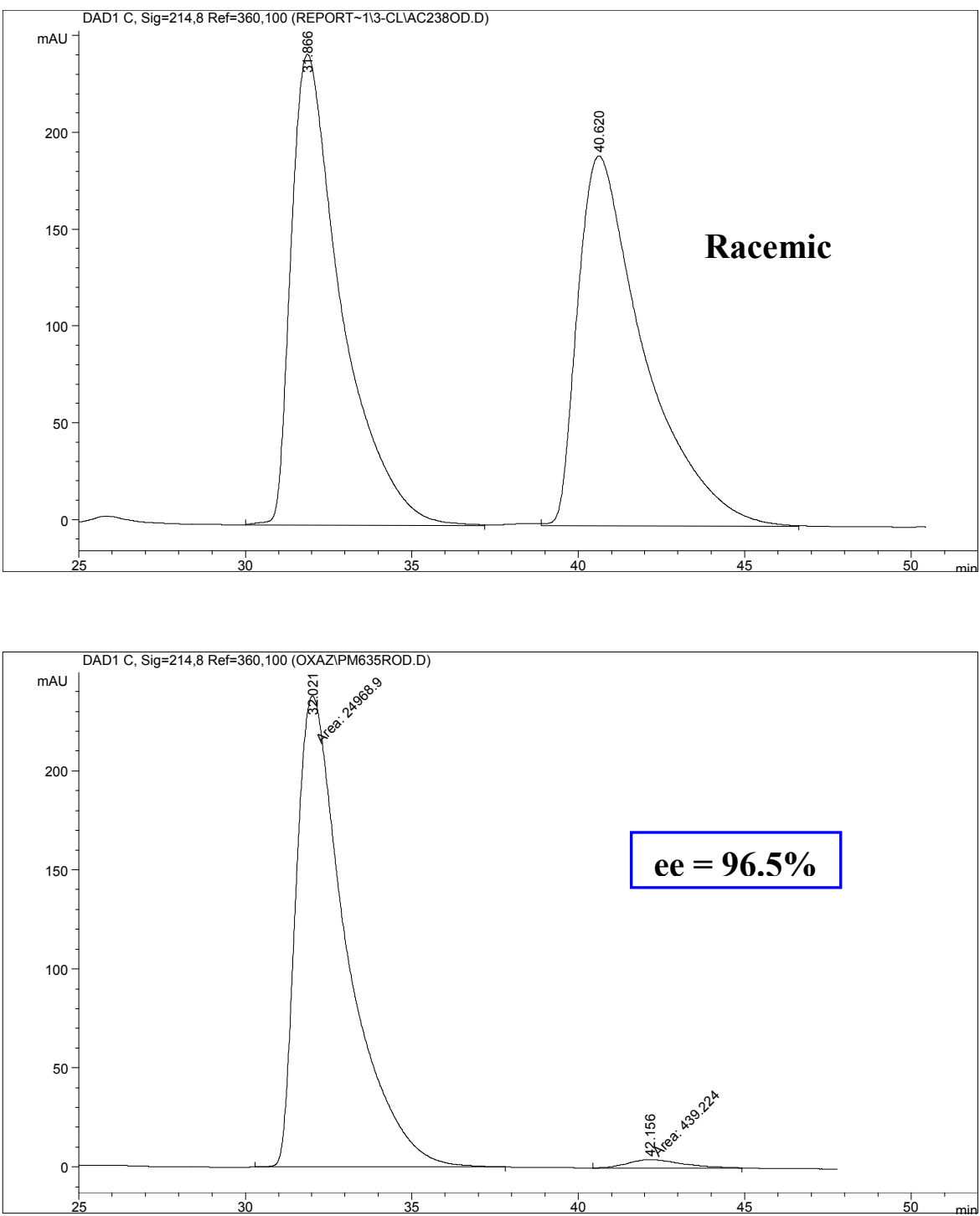

\begin{tabular}{ccc} 
Retention Times & Area & Area Percent \\
\hline 32.021 & $2.49689 \cdot 10^{4}$ & 98.2713 \\
42.156 & 439.22418 & 1.7287 \\
\hline Totals & $2.54081 \cdot 10^{4}$ &
\end{tabular}




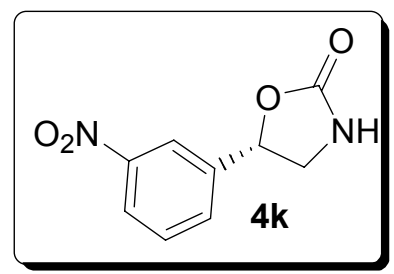

HPLC: CHIRALPAK OB-H 70/30 hexane / iso-propanol, 0.75 mL
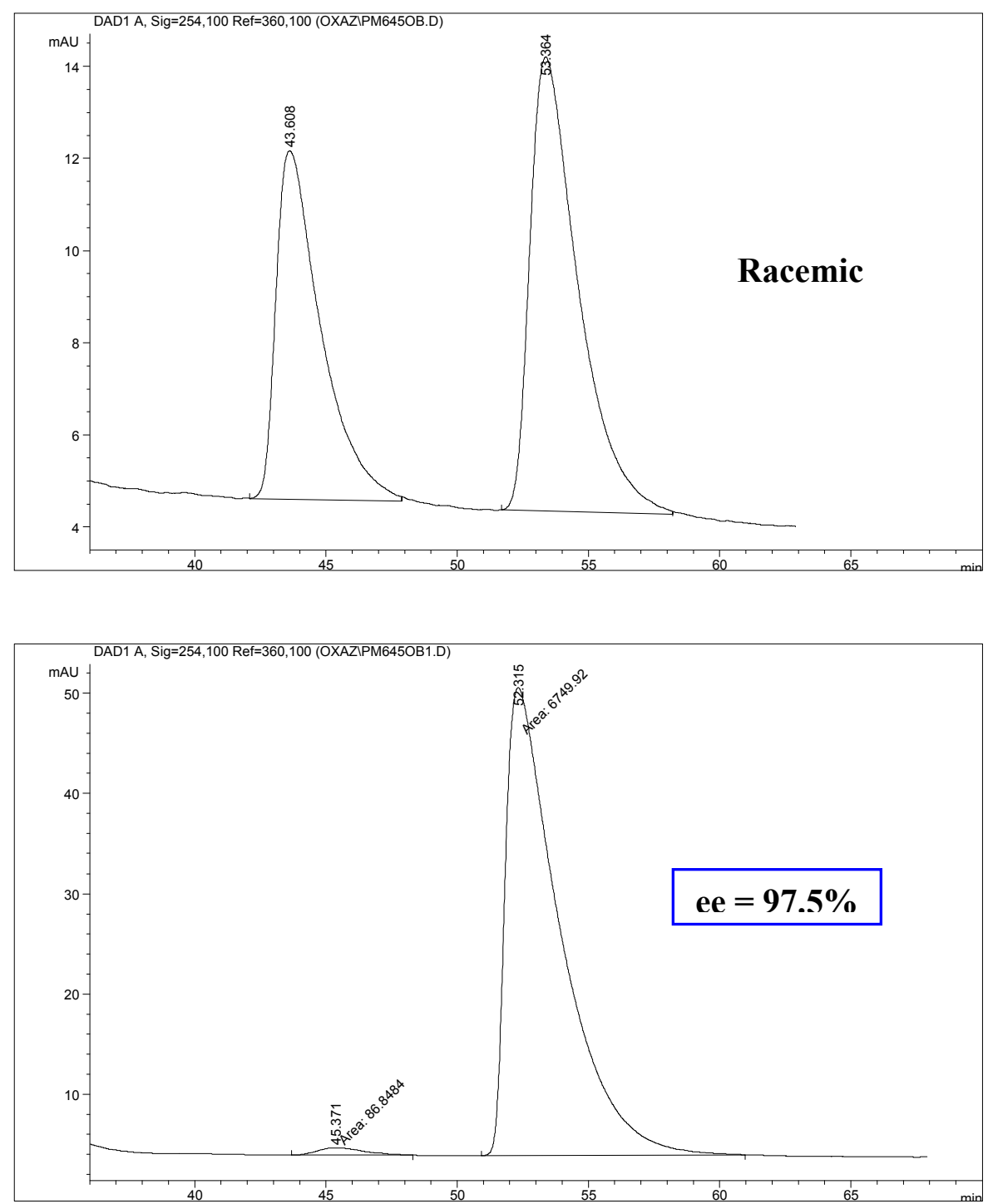

\begin{tabular}{ccc} 
Retention Times & Area & Area Percent \\
\hline 45.371 & 86.84843 & 1.2703 \\
52.315 & 6749.92480 & 98.7297
\end{tabular}

Totals

6836.77324 


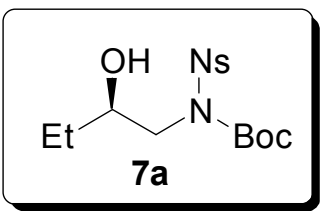

HPLC: CHIRALPAK AD-H 80/20 hexane / iso-propanol, $0.75 \mathrm{~mL}$
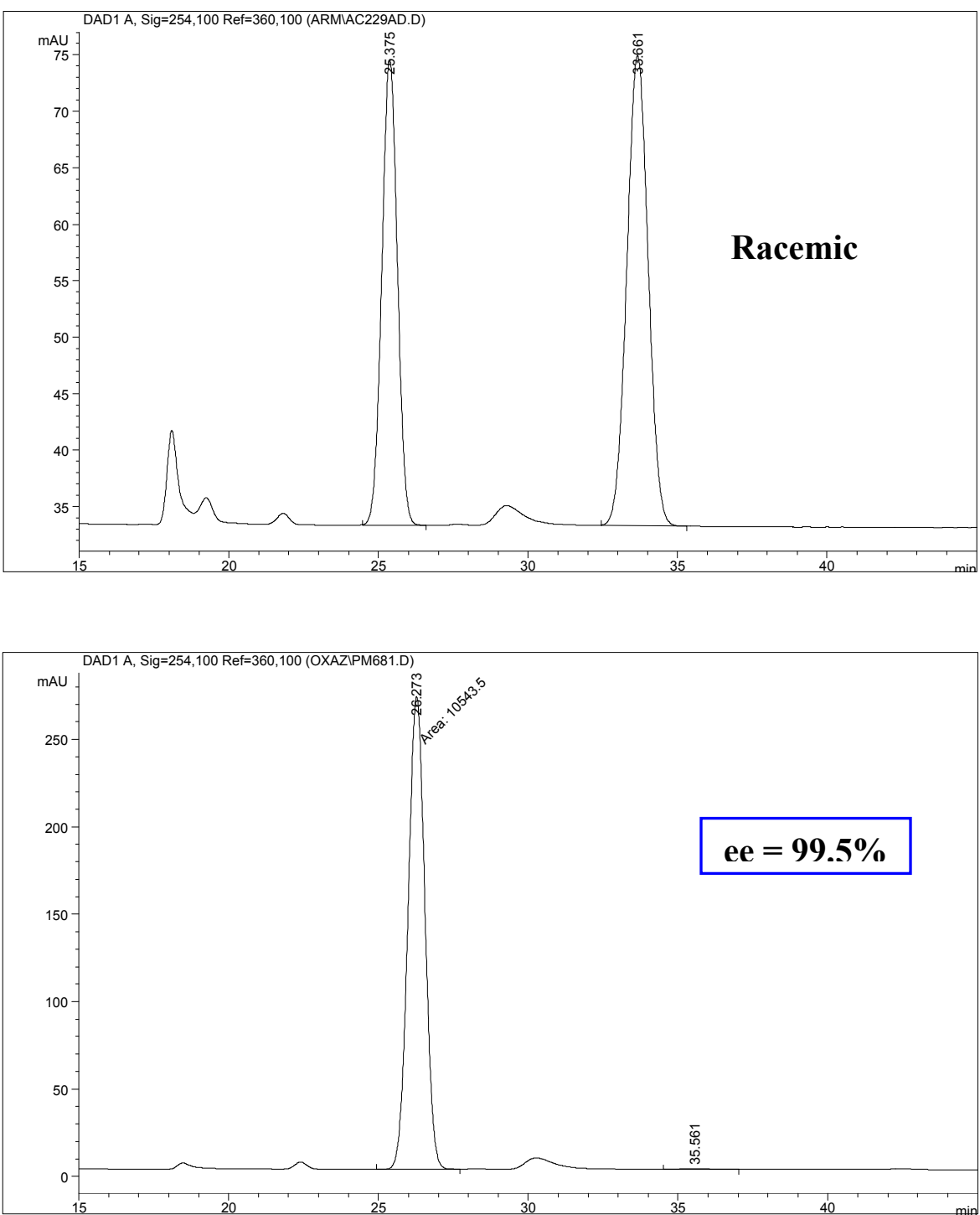

\begin{tabular}{ccc} 
Retention Times & Area & Area Percent \\
\hline \hline 26.273 & $1.05435 \cdot 10^{4}$ & 99.7414 \\
35.561 & 27.33446 & 0.2586 \\
\hline
\end{tabular}

Totals

$1.05709 \cdot 10^{4}$ 


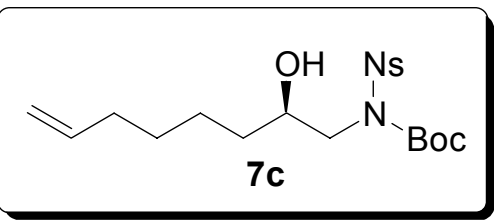

HPLC: CHIRALPAK AD-H 80/20 hexane / iso-propanol, 0.75 mL
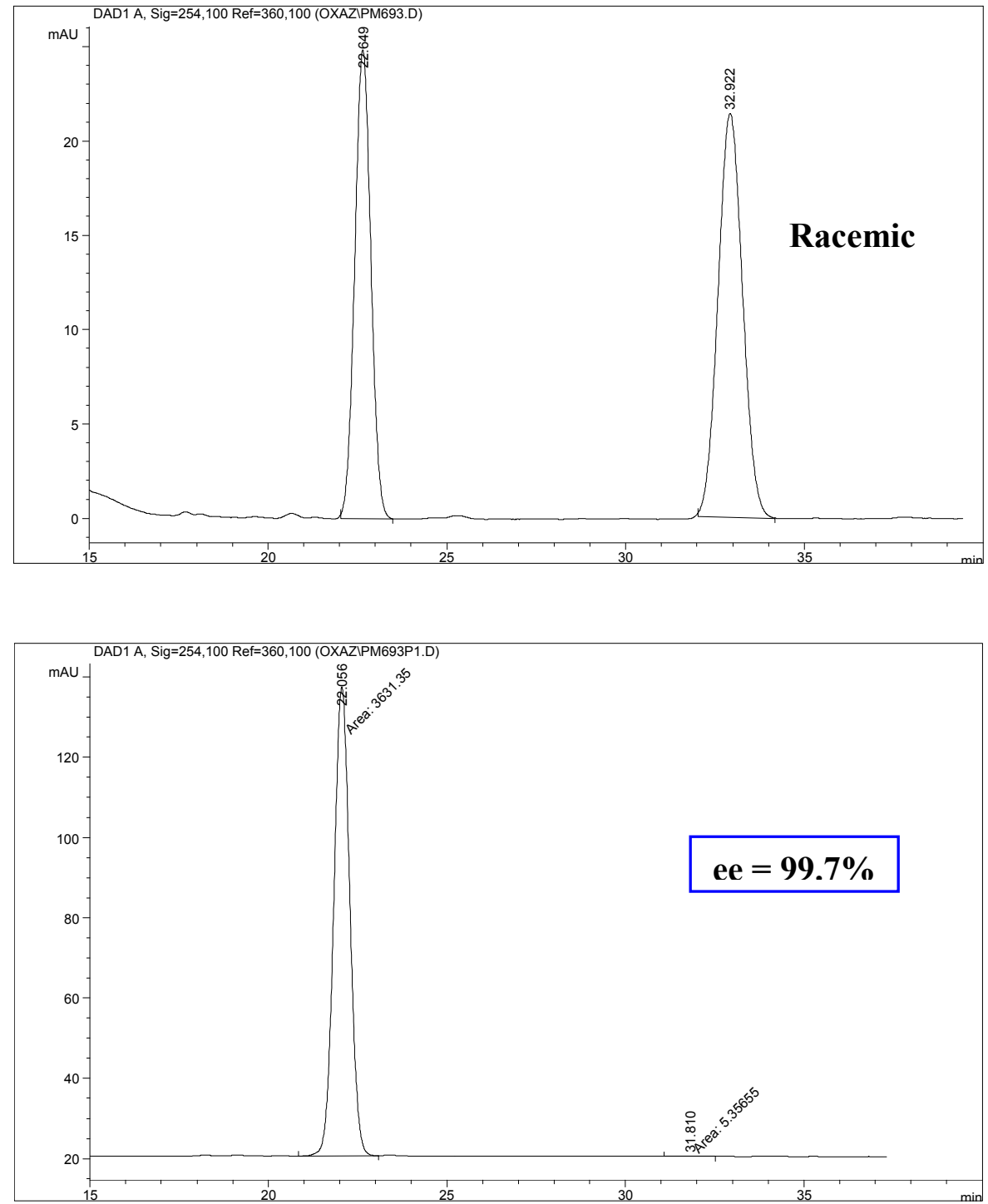

Retention Times

Area

Area Percent

\begin{tabular}{ccc}
\hline \hline 22.056 & 3631.35 & 99.853 \\
31.810 & 5.3565 & 0.147 \\
\hline
\end{tabular}

Totals

3636.70714 


\section{${ }^{1}$ H NMR Spectra}

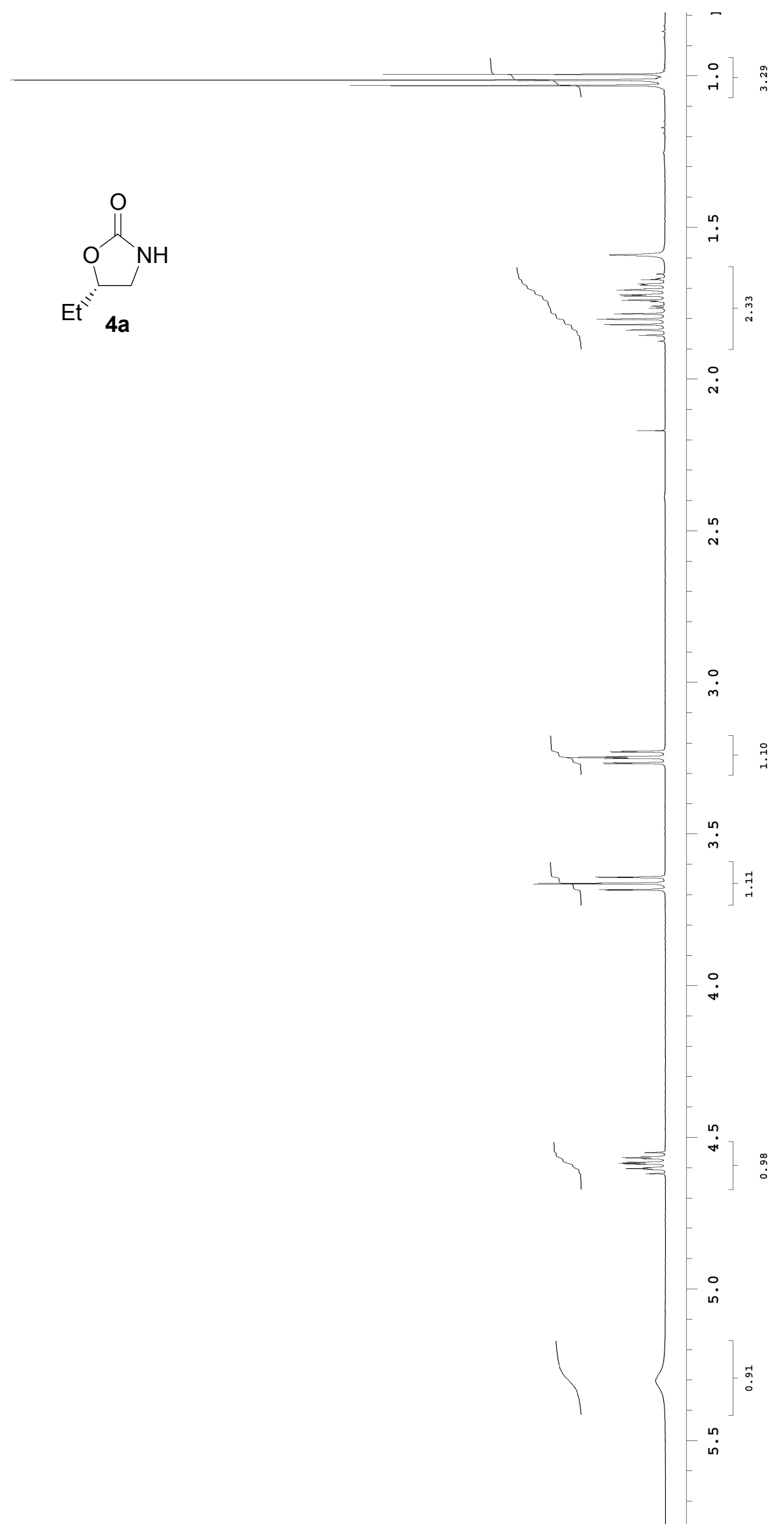




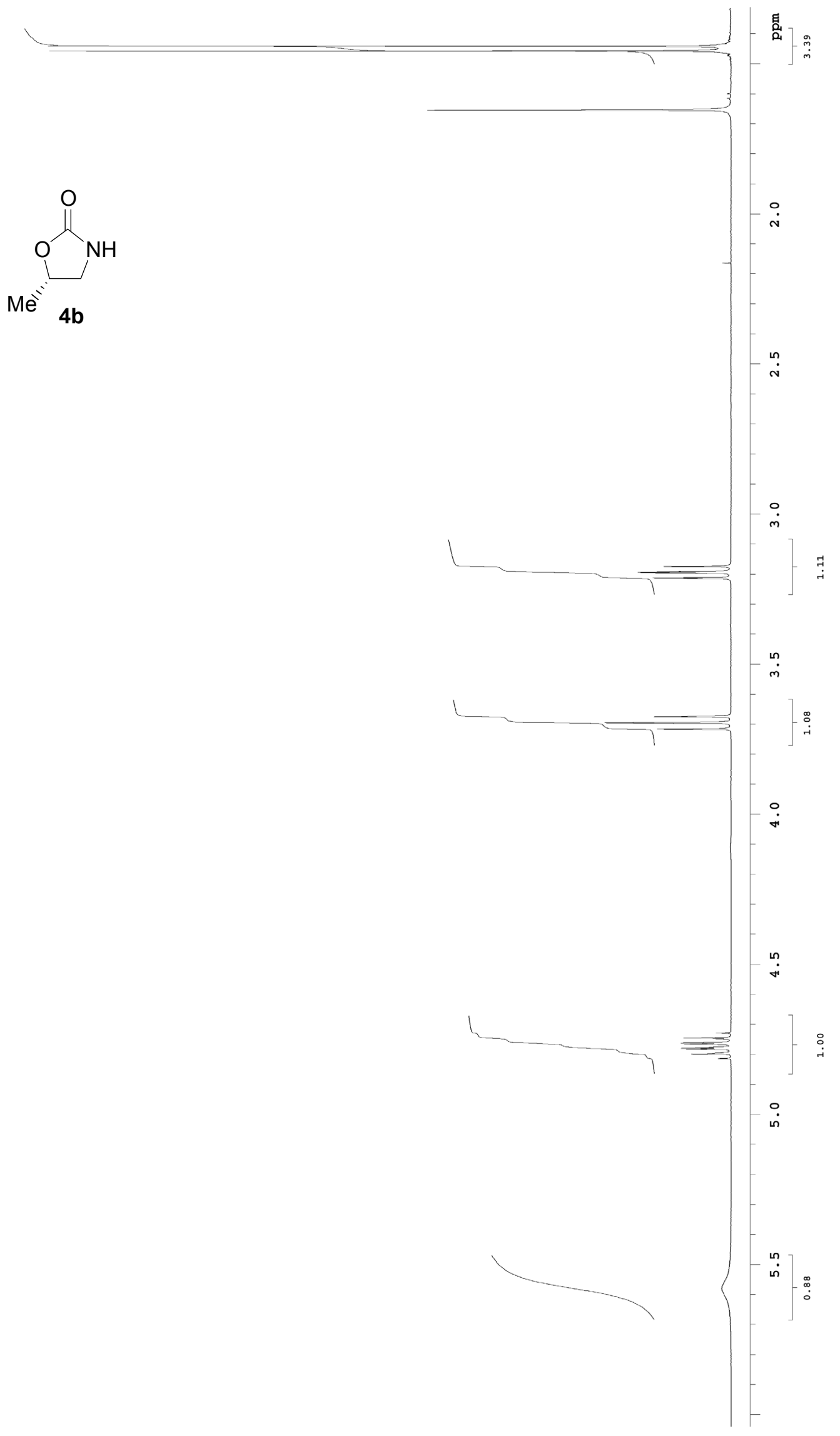




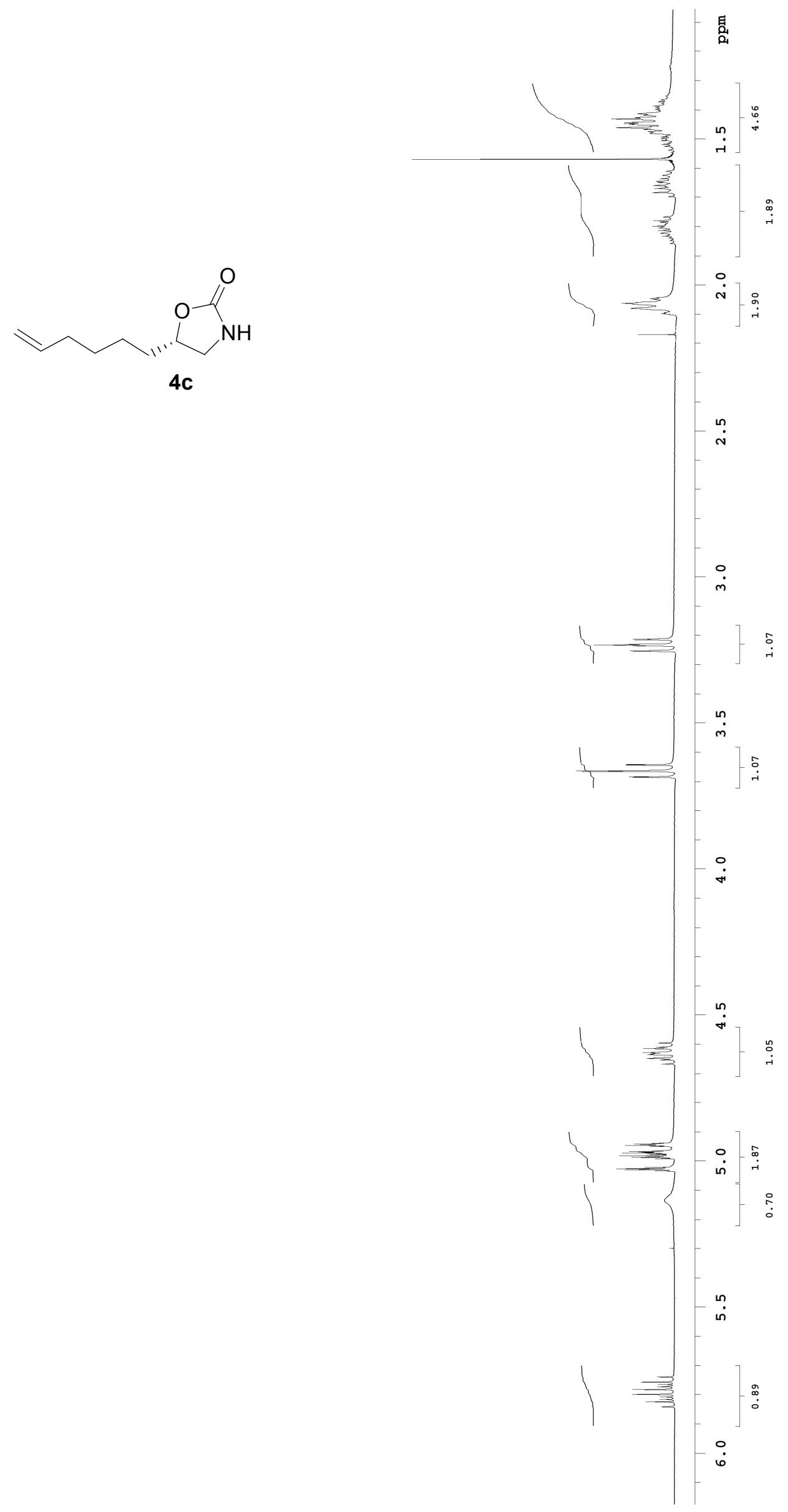



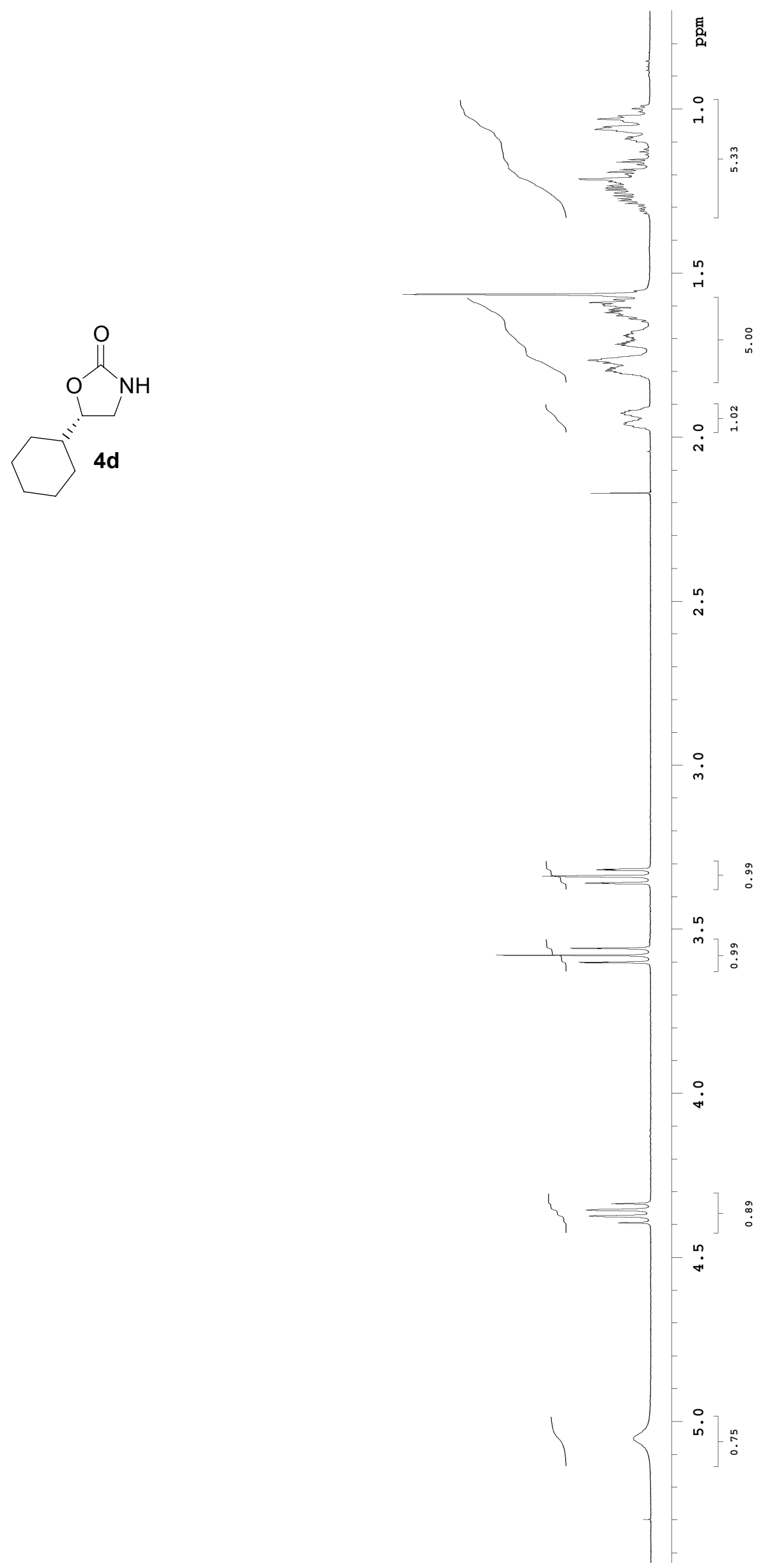


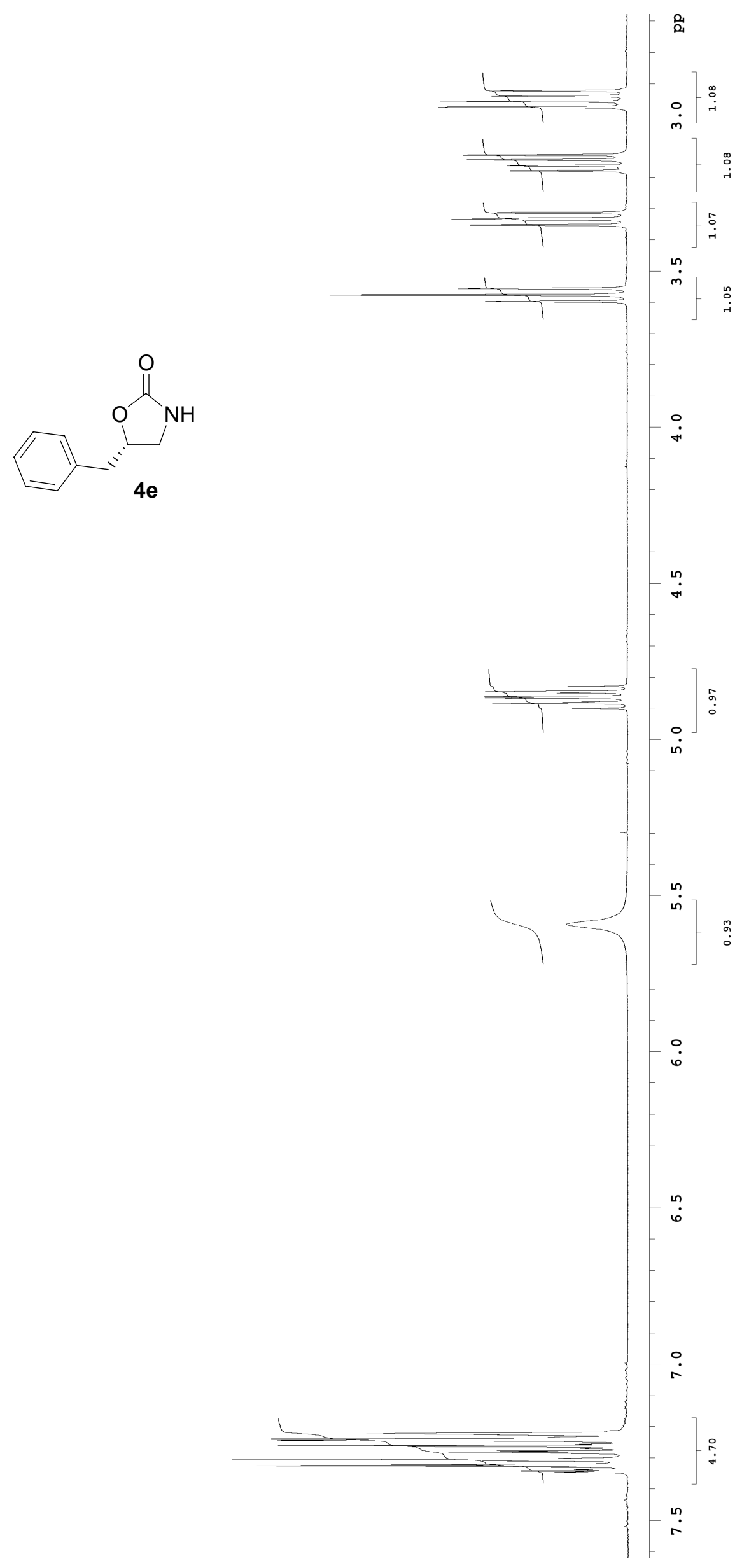




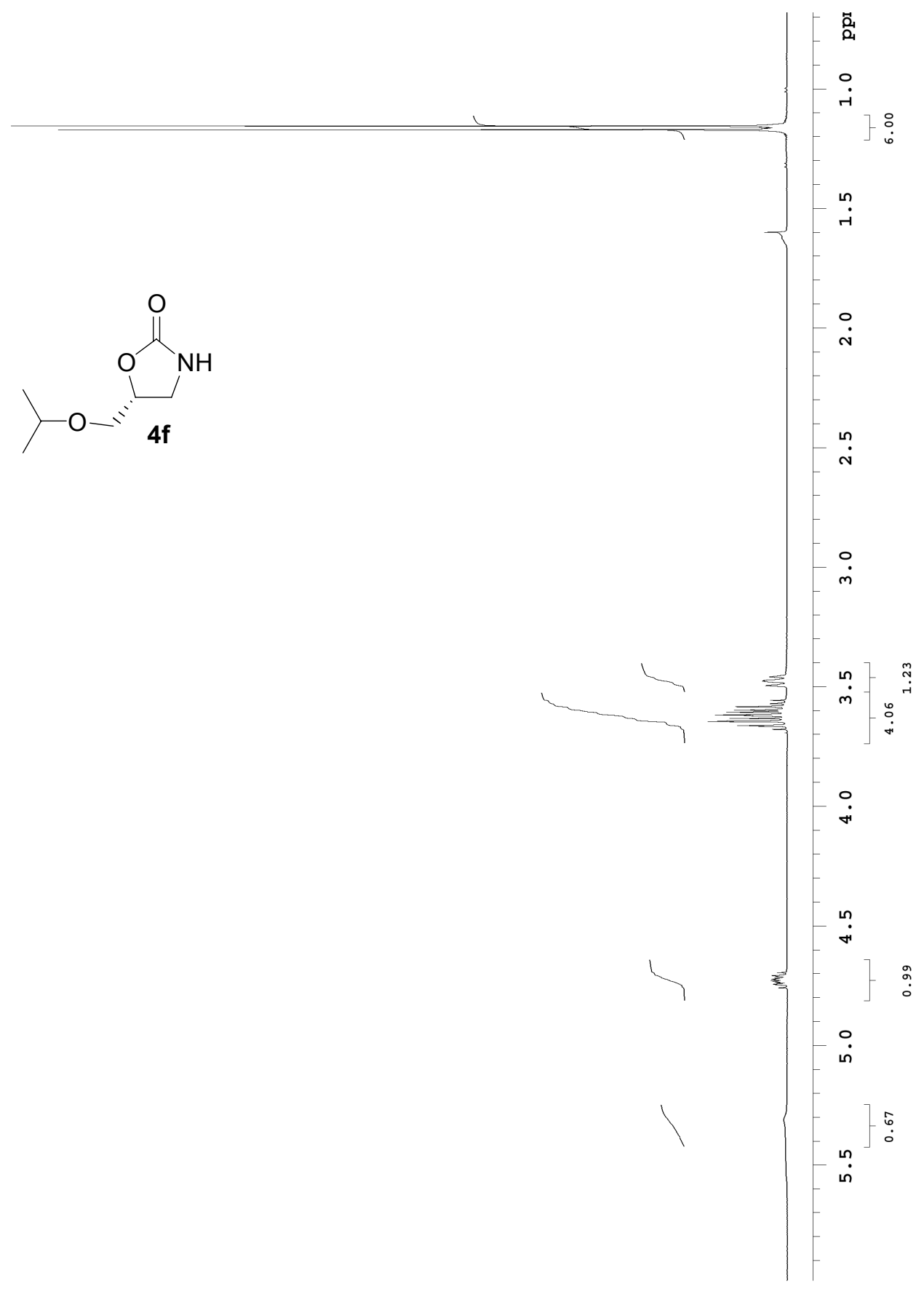




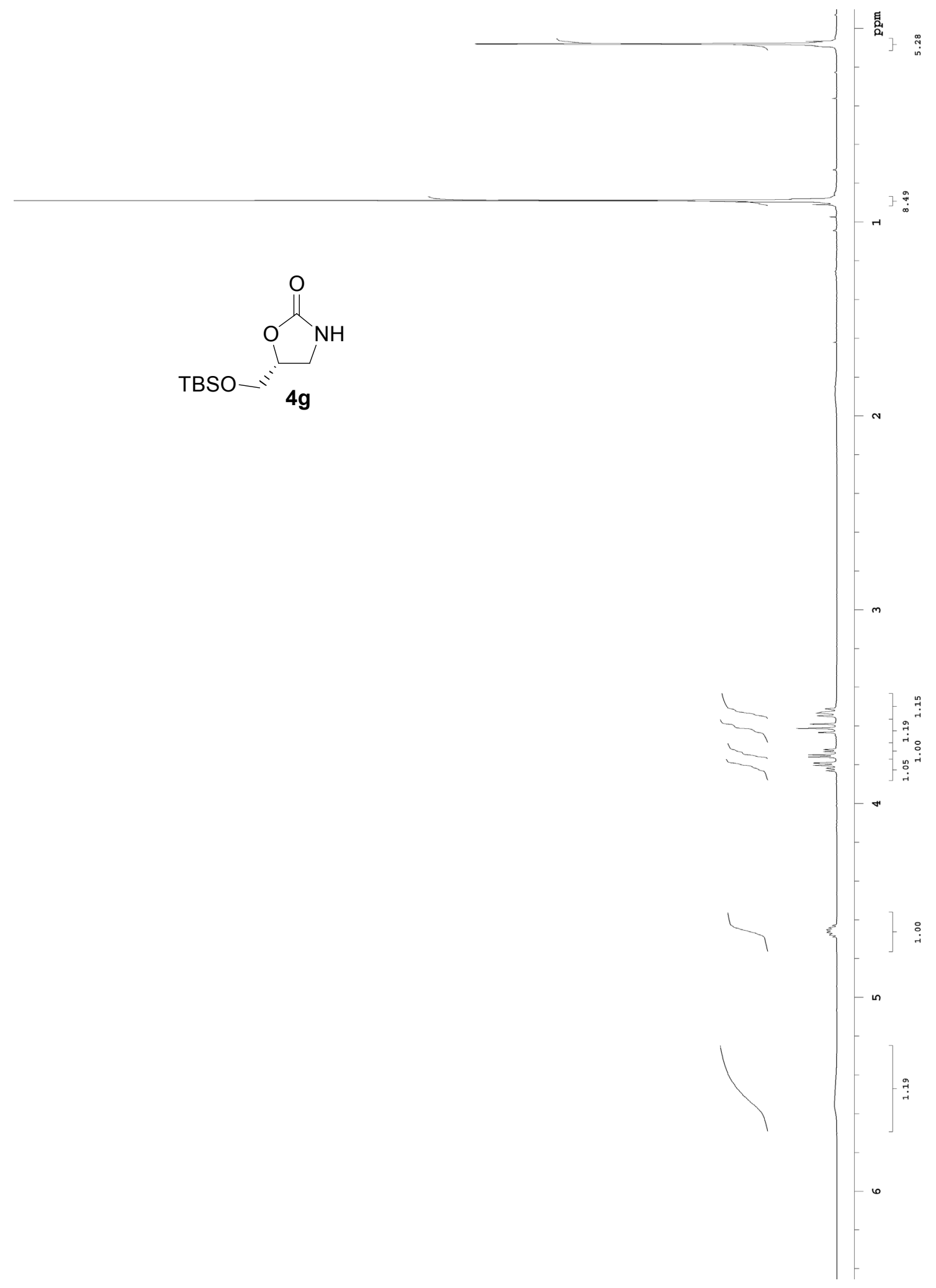




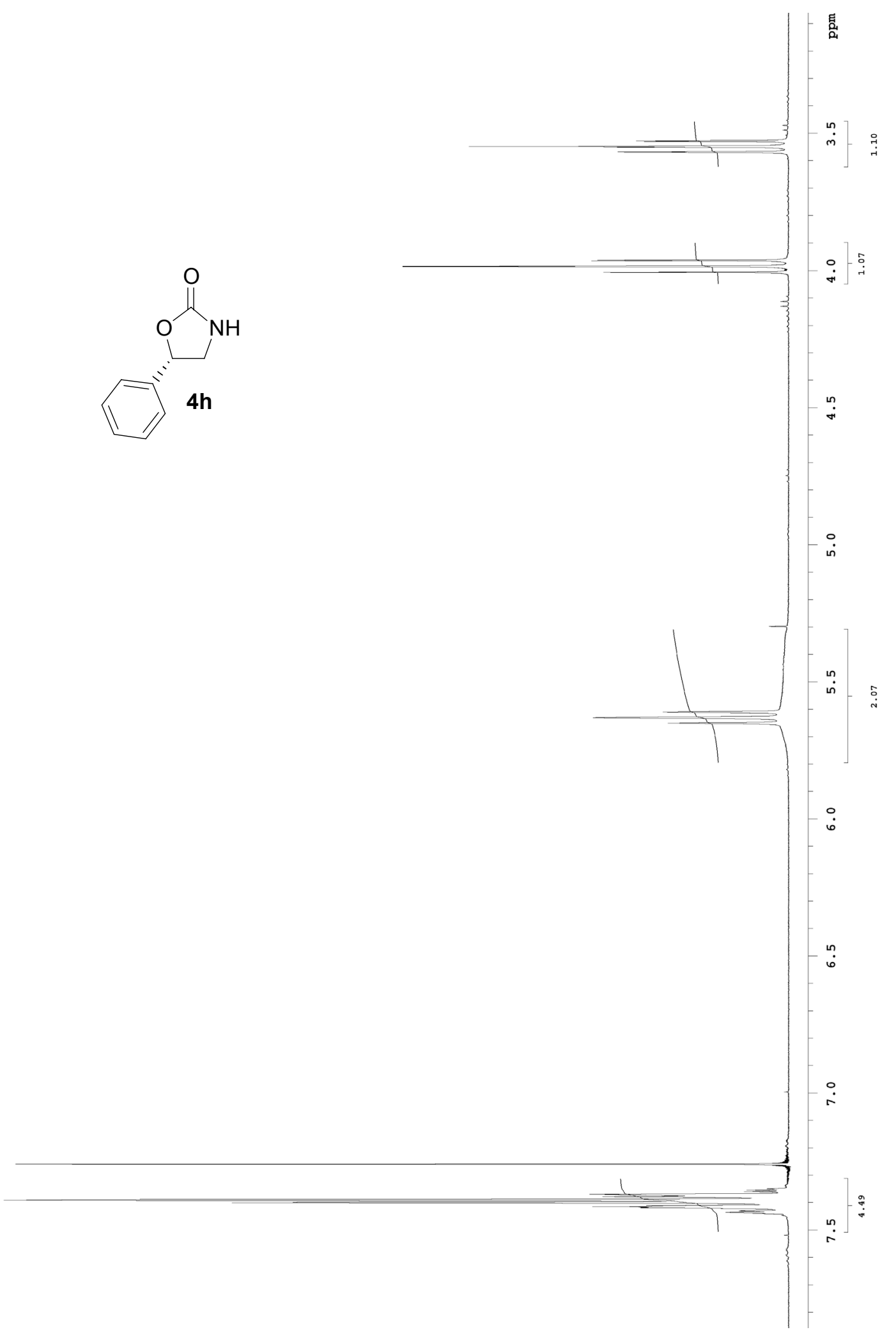




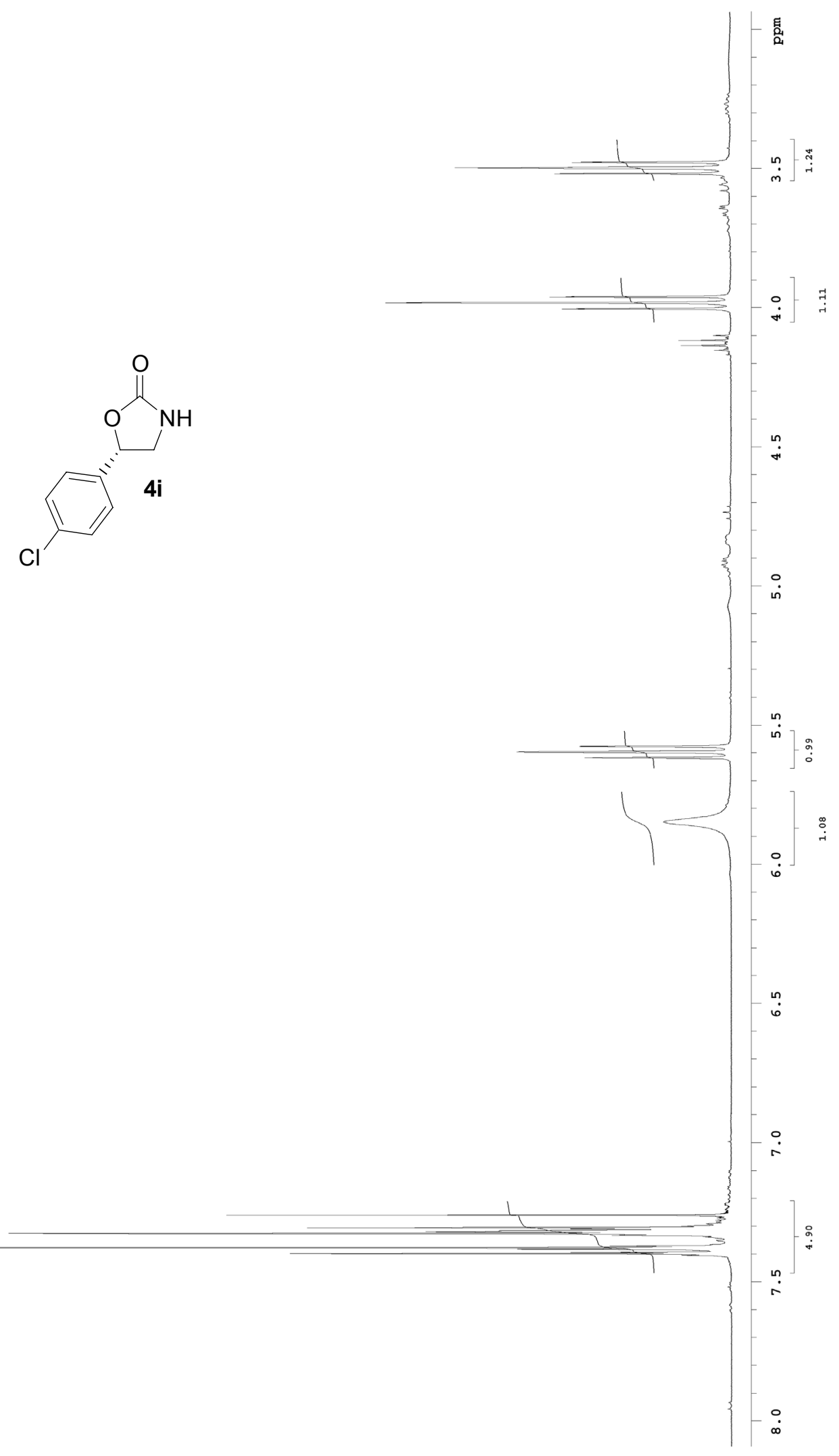




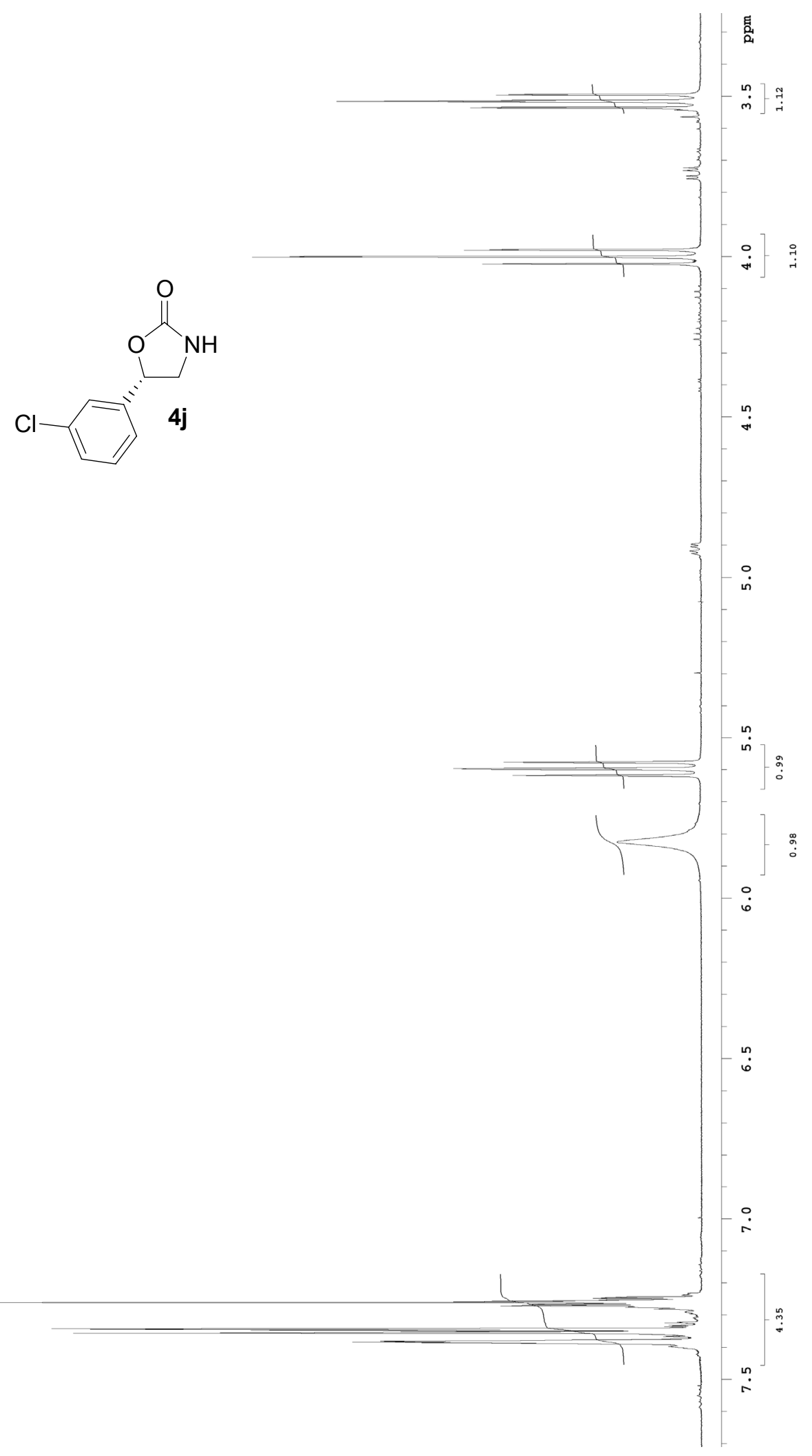




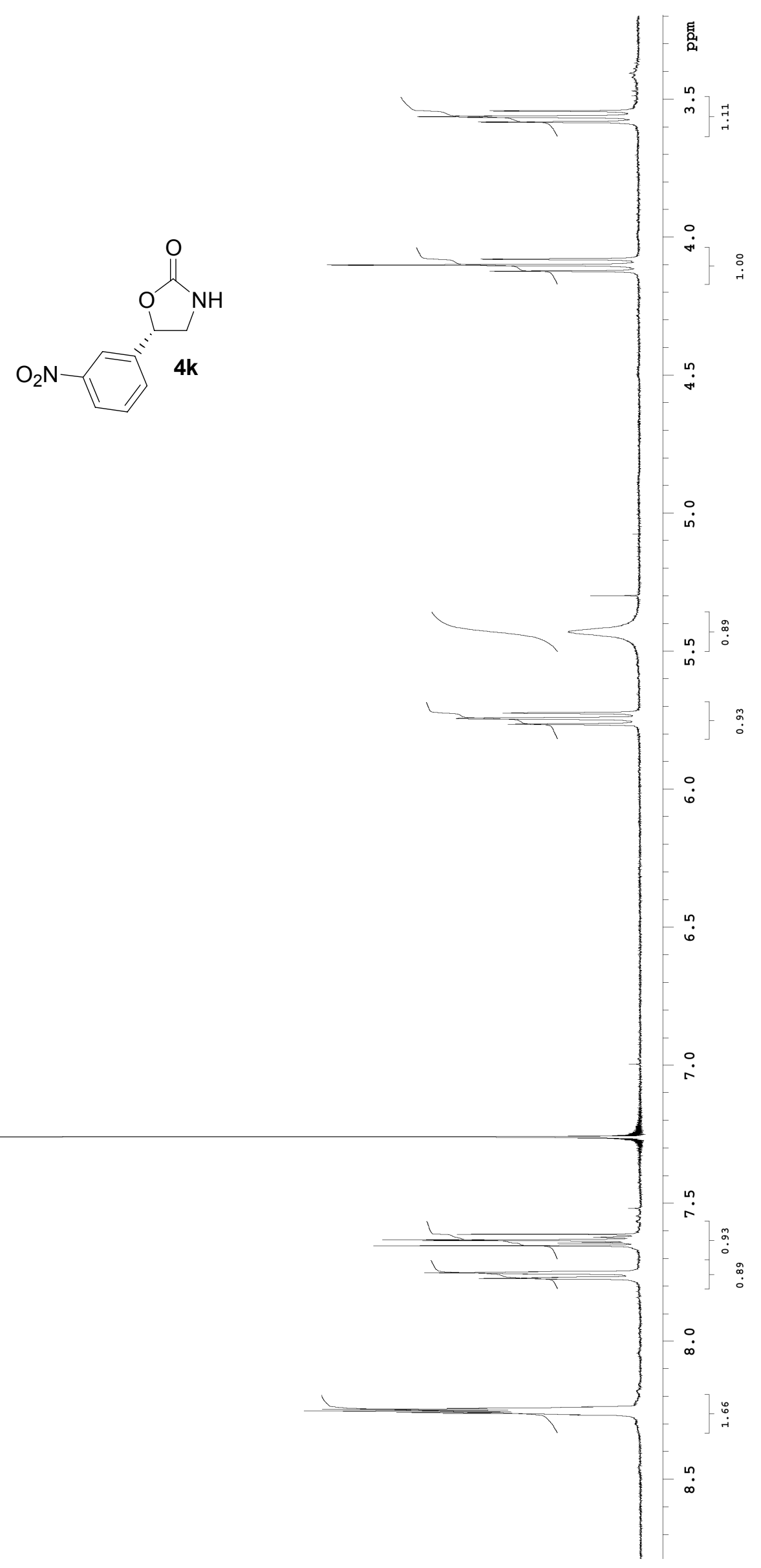




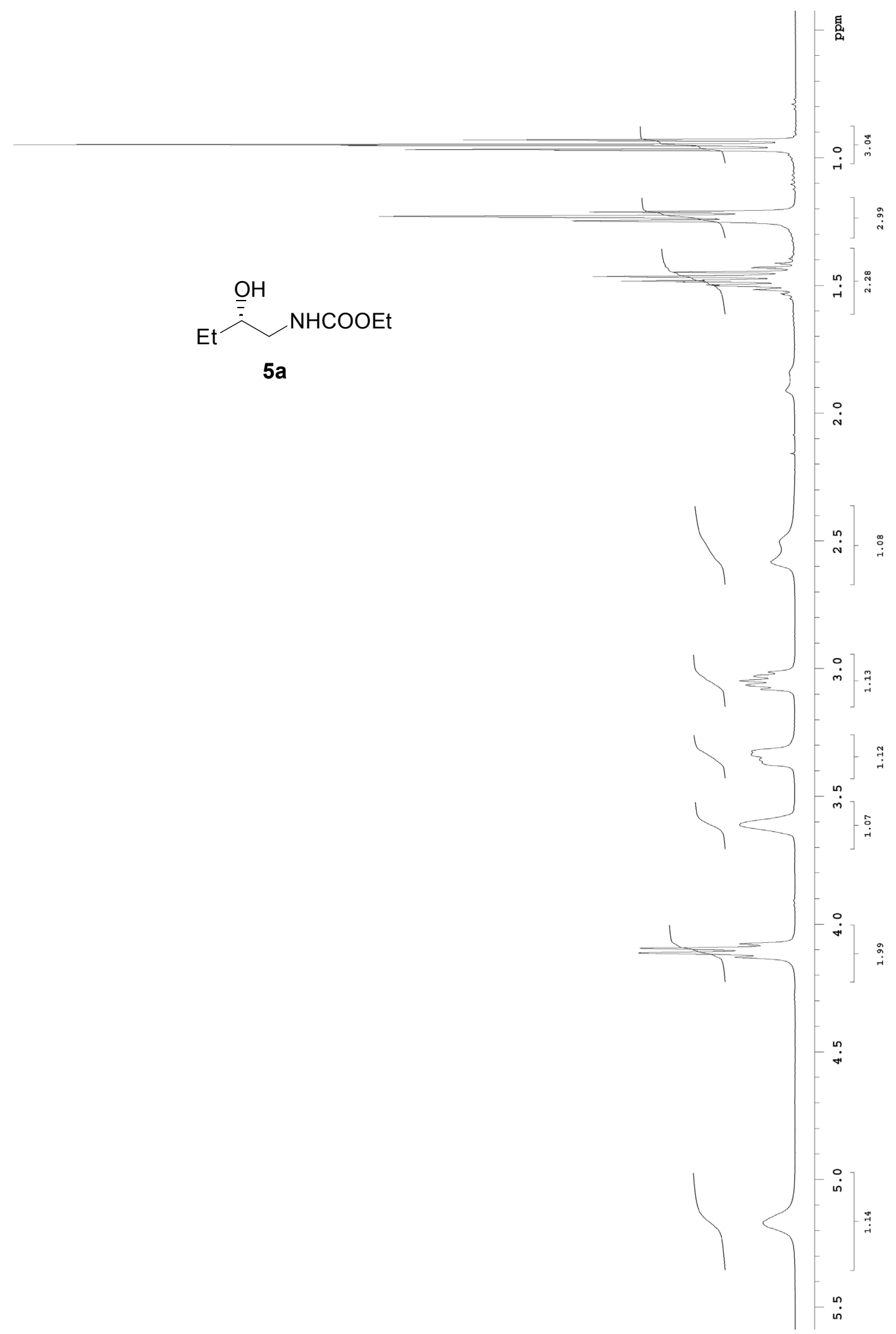




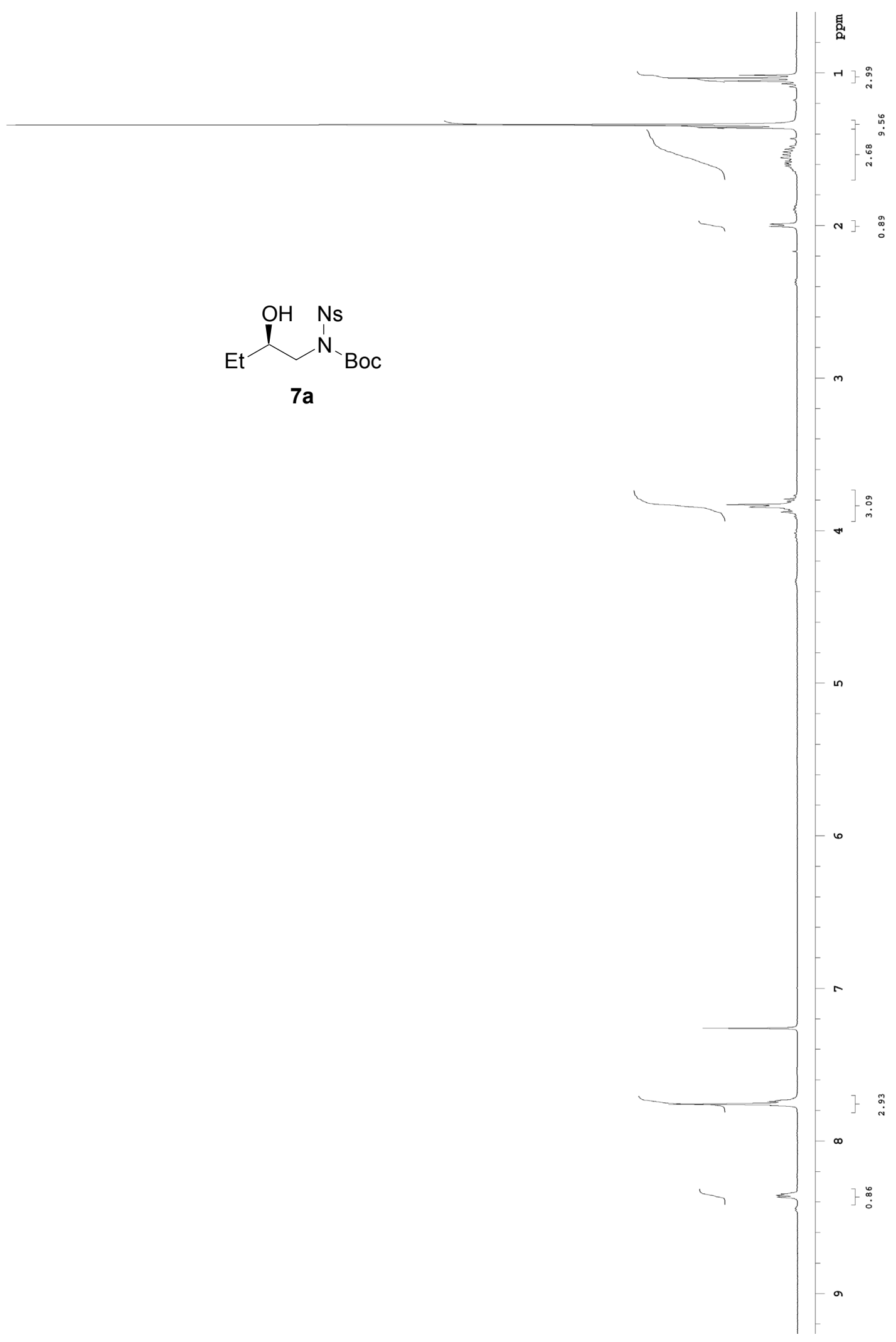




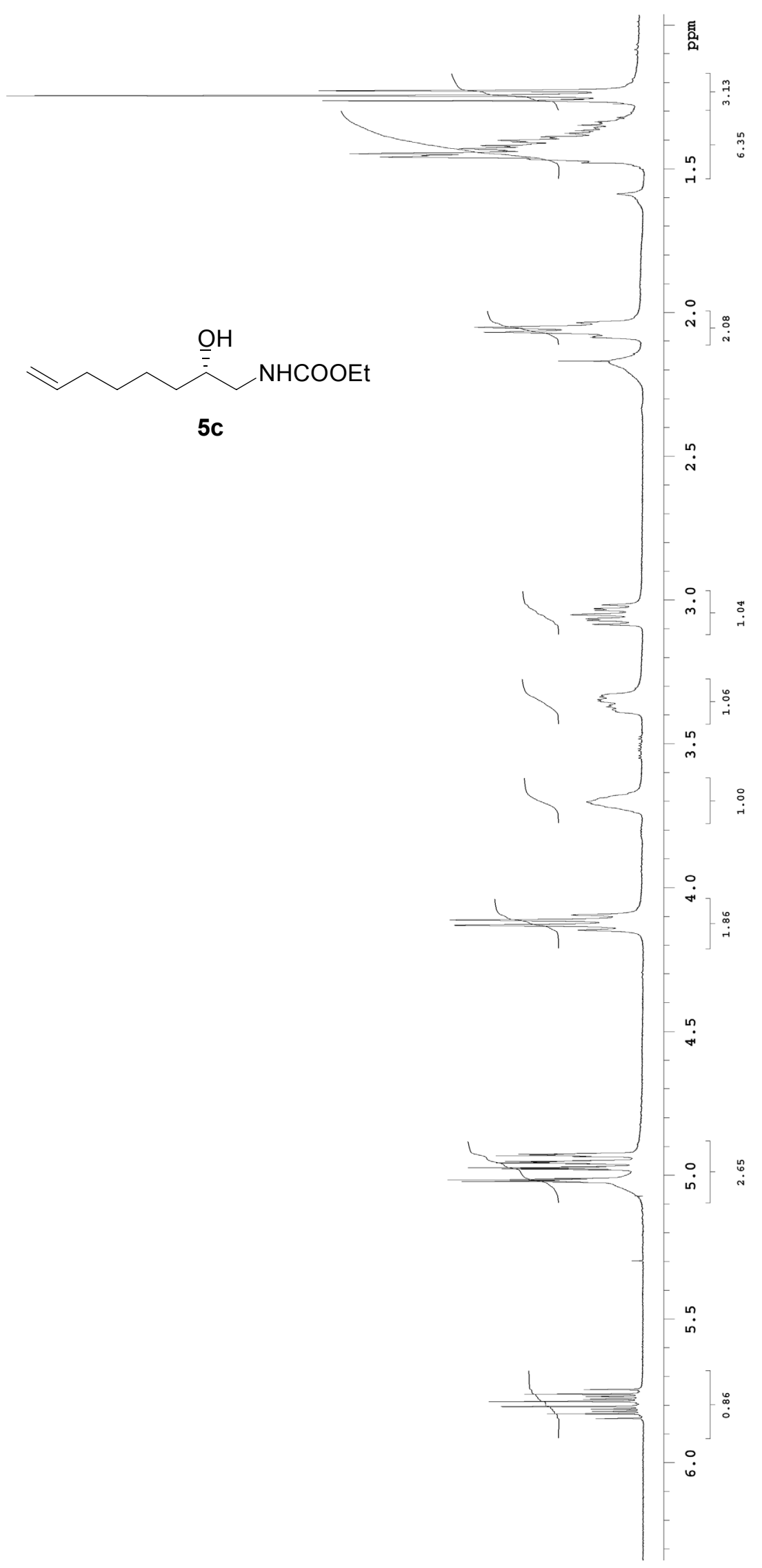




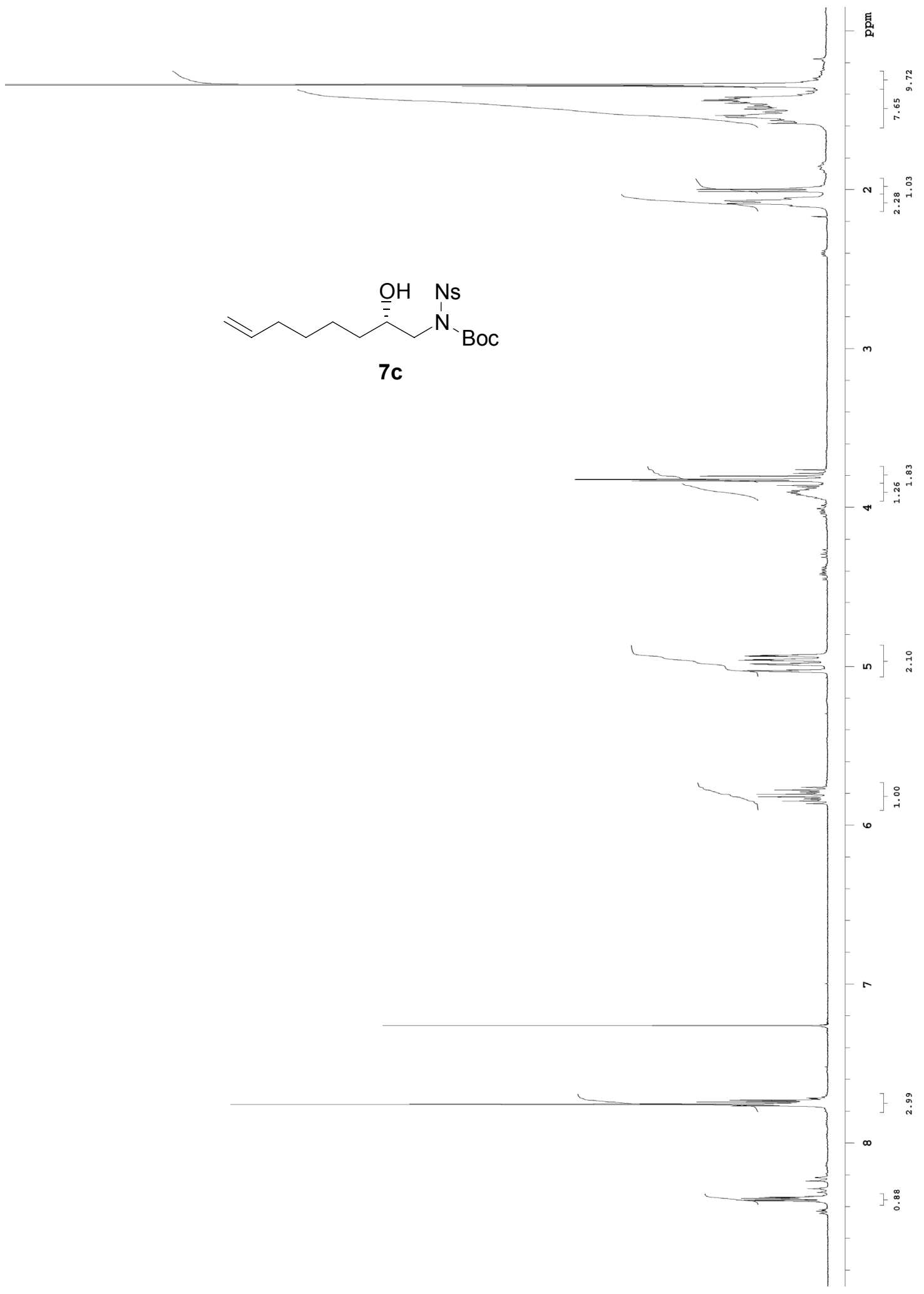

